\title{
Outflows, Accretion, and Clustered Protostellar Cores around a Forming O Star
}

\author{
Keping Qiu \\ Department of Astronomy, Nanjing University, Nanjing 210093, China \\ Harvard-Smithsonian Center for Astrophysics, 60 Garden Street, Cambridge, MA, USA \\ Max-Planck-Institute for Radioastronomy, Auf dem Hügel 69, 53121 Bonn, Germany \\ kqiu@mpifr-bonn.mpg.de \\ Qizhou Zhang \\ Harvard-Smithsonian Center for Astrophysics, 60 Garden Street, Cambridge, MA, USA \\ qzhang@cfa.harvard.edu \\ Karl M. Menten \\ Max-Planck-Institute for Radioastronomy, Auf dem Hügel 69, 53121 Bonn, Germany \\ kmenten@mpifr-bonn.mpg.de
}

\begin{abstract}
We present a Submillimeter Array study in the $1.3 \mathrm{~mm}$ waveband of the NGC 7538 IRS 1-3 massive star-forming region. The brightest core in the $\mathrm{mm}$ continuum map, MM1, harbors the IRS 1 young O star. The core has a gas temperature of about $245 \mathrm{~K}$ and shows spatially unresolved emission in complex organic molecules, all typical of a hot molecular core. Toward MM1, redshifted absorption is seen in molecular lines with different energies above the ground state. This absorption probes inward motion of the dense gas toward the central young $\mathrm{O}$ star, and the estimated mass accretion rate reaches $10^{-3} M_{\odot} \mathrm{yr}^{-1}$. Multiple outflows are seen in the $\mathrm{CO}$ and ${ }^{13} \mathrm{CO}$ maps. The gas mass of $50 M_{\odot}$ and mass outflow rate of $2.5 \times 10^{-3} M_{\odot} \mathrm{yr}^{-1}$ measured in CO line wings are dominated by the MM1 outflow, which is most likely driven by a fast wide-angle wind. Apart from MM1, we discover eight new dusty cores, MM2-9, within a projected distance of $0.35 \mathrm{pc}$ from MM1. These cores show no counterpart in infrared or radio continuum emission, while seven of them appear to be forming intermediate-
\end{abstract}


to high-mass stars. This manifests a deeply embedded star-forming component of the parent cloud of IRS 1-3. Apparently we are observing a Trapezium system in formation, and the system is presumably surrounded by a cluster of lower mass stars.

Subject headings: ISM: individual (NGC 7538) — ISM: jets and outflows - HiI regions — stars: formation — stars: early-type — techniques: interferometry

\section{Introduction}

NGC 7538 is a large, optically visible Hir region in the Perseus arm. About 2 arcmin (1.5 pc) southeast to the center of the Hir region is a site of active massive star formation known for three radio and infrared (IR) sources IRS 1-3 (Martin 1973; Wynn-Williams et al. 1974). Located at a distance of $2.65 \mathrm{kpc}$ (Moscadelli et al. 2009), the IRS 1-3 region has a total luminosity of $1.4 \times 10^{5} L_{\odot}$, of which the luminosity of IRS 1 is about $8 \times 10^{4} L_{\odot}$, equivalent to a ZAMS spectral type of O7.5 (Werner et al. 1979; Campbell \& Thompson 1984; Akabane \& Kuno 2005). IRS 2 and IRS 3 are two nearby sources less obscured than IRS 1. IRS 2 has a luminosity of $5 \times 10^{4} L_{\odot}$ and is associated with a compact HiI region; IRS 3 has a luminosity of $6 \times 10^{3} L_{\odot}$ and is associated with an ultracompact Hir region (Campbell \& Thompson 1984; Bloomer et al. 1998; Sandell et al. 2009).

IRS 1 has attracted numerous studies. Radio continuum observations show that the ionized gas has a double-lobed structure within the central $0 . / 2$ region and more extended halos at a 1" scale (Campbell 1984; Gaume et al. 1995; Sandell et al. 2009), and exhibits time variation in emission flux (Franco-Hernández \& Rodríguez 2004). Radio recombination lines (RRLs) observed at $\mathrm{cm}$ and $\mathrm{mm}$ wavelengths show extremely broad line widths, suggestive of expanding motion of the ionized gas (Gaume et al. 1995; Keto et al. 2008). In high-angular-resolution mid-IR images, a shell-like structure extending from IRS 1 to about $4^{\prime \prime}$ to the northwest is interpreted as dust emission on the wall of the outflow cavity (De Buizer \& Minier 2005). $K^{\prime}$-band images obtained with speckle interferometry show a fan-shaped nebula extending from IRS 1 toward the northwest, and the emission is predominantly attributed to scattered light from an outflow cavity (Kraus et al. 2006). On larger scales, single-dish CO observations reveal a bipolar outflow with a northwest-southeast (NWSE) orientation which is centered at IRS 1 (Kameya et al. 1989; Davis et al. 1998). IRS 1 is associated with various types of molecular masers (e.g., Pratap et al. 1989, and references therein). Pestalozzi et al. (2004) modeled a group of methanol masers detected toward IRS 1 as tracing an edge-on Keplerian disk around a central star of $30 M_{\odot}$, although the orientation of a possible disk around IRS 1 is controversial (e.g., De Buizer \& Minier 2005; 
Klaassen et al. 2009).

Existing high-angular-resolution observations toward NGC 7538 IRS 1 mostly focus on hot dust and ionized gas in the close vicinity of the young $\mathrm{O}$ star, while kinematics and physical conditions of molecular gas in the dusty envelope is less clear. Moreover, the molecular outflow in this region has not been observed at high angular resolutions; a pioneering interferometric study performed by Scoville et al. (1986) is of moderate resolution and image quality. In this paper we present a $1.3 \mathrm{~mm}$ waveband study of the NGC 7538 IRS 1 region using the Submillimeter Array 1 , aimed at understanding physical conditions and kinematics of the gas and dust cocoon surrounding the young $\mathrm{O}$ star and the nature of the molecular outflow. In its course we discovered clustered star-forming cores deeply embedded within the parent cloud of IRS 1-3 and multiple molecular outflows. A description of observations and data reduction is presented in Section 2. Observational results are presented in Section 3, followed by discussions on their implications in Section 4. Finally, a summary of the main findings is given in Section 5 ,

\section{Observations and Data Reduction}

\subsection{SMA Observations}

The SMA observations were undertaken on 2007 July 13 with eight antennas in the Compact-north configuration. The weather was very good during the observation, with 1.5 to $2.5 \mathrm{~mm}$ precipitable water vapor (PWV), corresponding to atmospheric optical depth at $225 \mathrm{GHz}, \tau_{225 \mathrm{GHz}}$, around 0.08 to 0.12 . To cover the entire outflow we observed two fields centered at (R.A., decl. $)_{\mathrm{J} 2000}=\left(23^{\mathrm{h}} 13^{\mathrm{m}} 43.75,61^{\circ} 28^{\prime} 21 .^{\prime \prime} 5\right)$ and $\left(23^{\mathrm{h}} 13^{\mathrm{m}} 46.75,61^{\circ} 27^{\prime} 59 .^{\prime \prime} 5\right)$, guided by previous single-dish observations (Davis et al. 1998). The $2 \times 2 \mathrm{GHz}$ correlator was configured to cover rest frequencies about 219.2 to $221.2 \mathrm{GHz}$ in the lower sideband and about 229.2 to $231.2 \mathrm{GHz}$ in the upper sideband, with a uniform spectral resolution of $812.5 \mathrm{kHz}\left(\sim 1.1 \mathrm{~km} \mathrm{~s}^{-1}\right)$. We observed 3c273 and Callisto for bandpass calibration and MWC349, for which a flux of $1.65 \mathrm{Jy}$ at $225 \mathrm{GHz}$ was adopted, for flux calibration. Time dependent gains were monitored by interleavingly observing J0102+584 and BL Lac every 30 mins. The primary calibrations were carried out with the IDL MIR package, which allows to determine antenna gains (versus frequency or time) by simultaneously fitting multiple calibrators. The calibrated visibilities were exported to MIRIAD for further processing. A

\footnotetext{
${ }^{1}$ The Submillimeter Array is a joint project between the Smithsonian Astrophysical Observatory and the Academia Sinica Institute of Astronomy and Astrophysics and is funded by the Smithsonian Institution and the Academia Sinica.
} 
continuum database was constructed from line-free channels using the MIRIAD task UVLIN. We self-calibrated the continuum solving for phase corrections with decreasing time intervals (5, 2, $1 \mathrm{~min})$, and then solving for phase and amplitude corrections for the last iteration. The solutions from the self-calibration were applied to spectral line data as well.

In Figure 1, the predominant core MM1 is more than 10 times brighter than MM2-9. As the dynamical range of an interferometric image is limited by incomplete $(u, v)$ sampling, we assess the reliability of MM2-9. We imaged J0102+584 and BL Lac and found that each of the two quasars appears as a point source at the phase center, and the position agrees with the nominal coordinate to $<0.1^{\prime \prime}$. We also found that features associated with MM2-9 are identifiable in the continuum and spectral line images without self-calibration. Furthermore, we obtained a model of MM1 by restricting CLEAN (a deconvolution task in MIRIAD) to a polygon closely around MM1, subtracted "MM1" from the calibrated visibilities using the task UVMODEL, and imaged the residual visibilities; MM1 was completely invisible while MM2-9 were clearly seen in the image, indicating that none of the fainter cores was falsely generated due to sidelobes of MM1 during deconvolution.

The final maps have a synthesized beam of $\sim 3^{\prime \prime} \times 2^{\prime \prime}$, and r.m.s. sensitivities of $\sim 5 \mathrm{mJy}$ for continuum and $\sim 35$ mJy per $1.2 \mathrm{~km} \mathrm{~s}^{-1}$ for lines.

\subsection{Short Spacings in $\mathrm{CO}$ and ${ }^{13} \mathrm{CO}(2-1)$}

In an interferometric observation, structures more extended than $1.2 \lambda / b_{\text {min }}$, where $\lambda$ is the observing wavelength and $b_{\text {min }}$ the shortest length of the projected baselines, cannot be effectively sampled. This filtering effect is significant for $\mathrm{CO}$ and ${ }^{13} \mathrm{CO}$ maps, particularly at velocities around the cloud velocity. Adding in single-dish observations is a way to overcome this effect (e.g., Qiu et al. 2009). For CO (2-1), we combined the SMA data with data obtained from the James Clerk Maxwell Telescope (JCMT) by Davis et al. (1998). For ${ }^{13} \mathrm{CO}(2-1)$, we carried out on-the-fly mapping observations with the Caltech Submillimeter Observatory 2 (CSO) $10.4 \mathrm{~m}$ telescope on 2008 June 11. During the last observation, the weather was reasonably good with about $4 \mathrm{~mm} \mathrm{PWV}$ and $\tau_{225 \mathrm{GHz}}$ around 0.2 . We obtained a $13 \times 13$ grid map centered on (R.A., decl. $)_{\mathrm{J} 2000}=\left(23^{\mathrm{h}} 13^{\mathrm{m}} 43.8,61^{\circ} 28^{\prime} 22 !^{\prime \prime} 0\right)$. The grid cell spacing is $10^{\prime \prime}$, roughly $1 / 3$ of the FWHM beam at this frequency. The effective integration time toward each cell is about $10 \mathrm{~s}$. The AOS spectrometer has 1024 channels throughout the $50 \mathrm{MHz}$ bandwidth, thus a spectral resolution of $48.8 \mathrm{kHz}$ (or $0.066 \mathrm{~km} \mathrm{~s}^{-1}$ ). The data were reduced using the standard CLASS package. The combination of the SMA and single-dish

\footnotetext{
${ }^{2}$ The Caltech Submillimeter Observatory is supported by the NSF grant AST-0229008
} 
data was conducted in MIRIAD following a procedure outlined in Zhang et al. (1995). The final $\mathrm{CO}$ and ${ }^{13} \mathrm{CO}$ maps were smoothed to $2 \mathrm{~km} \mathrm{~s}^{-1}$ per channel.

\section{Results}

\section{1. $\quad 1.3 \mathrm{~mm}$ Continuum Emission}

Figure 1 presents the $1.3 \mathrm{~mm}$ continuum map, which reveals a cluster of nine $\mathrm{mm}$ cores, namely MM1 to MM9 in order of peak fluxes. Measured parameters of these cores are listed in Table 1. The brightest core MM1 coincides with the luminous IR and radio source IRS 1. The $1.3 \mathrm{~mm}$ continuum flux has a contribution of $\sim 1.5 \mathrm{Jy}$ from the free-free emission extrapolated from cm measurements (Sandell et al. 2009), and the dust emission flux is about 2.1 Jy. The $1.3 \mathrm{~mm}$ emission from MM2 to MM9 is entirely dominated by the dust emission, since none of them is seen in $\mathrm{cm}$ continuum. In contrast to IRS 1 , neither IRS 2 nor IRS 3 appears to be embedded within a dense dusty core.

Assuming thermal equilibrium between the dense gas and dust, we adopt a temperature of $245 \mathrm{~K}$ for MM1 derived from a local thermodynamical equilibrium (LTE) model of the $\mathrm{CH}_{3} \mathrm{CN}$ emission (Section 3.3.1). Cores MM2-9 have much lower temperatures; we estimate temperatures from $\mathrm{NH}_{3}$ data (Zheng et al. 2001) for MM6-9 (Section 3.3.2) and adopt an averaged temperature of $40 \mathrm{~K}$ for MM2-5 (Section 4.3). We then estimate gas masses of all the

cores by adopting the dust opacity $\kappa_{225 \mathrm{GHz}}=1 \mathrm{~cm}^{2} \mathrm{~g}^{-1}$ (Ossenkopf \& Henning 1994), which is equivalent to an opacity index $\beta$ of 1.375 for $\kappa_{\nu}=10\left(\frac{\nu}{1.2 \mathrm{THz}}\right)^{\beta} \mathrm{cm}^{2} \mathrm{~g}^{-1}$ (Hildebrand 1983), and a canonical gas-to-dust mass ratio of 100. The derived core masses are listed in Table 1 . The error bars of the core masses presented in Table 1 account for errors in measuring the continuum fluxes and calculating the temperatures. For MM1, the uncertainty in extrapolating the free-free emission is also taken into account. For MM2-5, an averaged uncertainty of $35 \%$ is assumed for the adopted temperature. There is a systemic uncertainty, $9 \%$, introduced by the distance error (0.12 kpc, Moscadelli et al. 2009). Other uncertainties come from possible variations of the gas-to-dust mass ratio and the dust opacity; the latter can be constrained with future high angular resolution observations at different wavelengths.

\subsection{Molecular Outflows}

Figure 2 shows the velocity integrated CO (2-1) emission obtained from the combined SMA and JCMT data sets. Velocity channel maps of the emission are shown in Figure 3 , Overall the CO emission shows spatially separated blue- and redshfited lobes in a NW-SE 
orientation, whereas the detailed structures are ascribed to multiple outflows rather than a simple bipolar outflow seen in previous single-dish observations.

In the integrated map, the blueshifted lobe appears as a curved filament originating from MM1 and diverging into three "branches" toward the northwest, north, and northeast. However, from the orientation, the northeastern branch with a position angle (PA) of about $40^{\circ}$ is more likely arising from MM4 or IRS 3; from the channel maps at -78 to -64 $\mathrm{kms}^{-1}$, the northern branch with a PA of about $0^{\circ}$ may originate from IRS 3 or MM4 as well. In addition, at least four more dusty cores, MM2, MM6, MM7, and MM8 appear to be associated with blueshifted CO outflows: an elongated feature extending from MM6 to the southeast is seen in the integrated map and blueshifted channel maps of $\geq-78 \mathrm{~km} \mathrm{~s}^{-1}$; emission around MM8 and extending to the northwest is seen in the integrated map and blueshifted channel maps of $\geq-74 \mathrm{~km} \mathrm{~s}^{-1}$; emission associated with MM2 is seen as a knot to its immediate southwest at -78 to $-72 \mathrm{~km} \mathrm{~s}^{-1}$ and as a compact elongation at -70 to $-68 \mathrm{~km} \mathrm{~s}^{-1}$; compact and faint emission extending from MM7 to the southwest is visible at -70 to $-66 \mathrm{kms}^{-1}$.

The redshifted emission appears as a large-scale "arc" in the integrated map, while the detailed structures can be attributed to at least two cores, MM1 and MM7. MM1 is presumably the powering source of the emission extending from around MM1 to a bright clump about $20^{\prime \prime}(0.26 \mathrm{pc})$ to the southeast; the clump is encompassed by a C-shaped structure seen in $\mathrm{C}^{18} \mathrm{O}(2-1)$. MM7 is likely the central source of the $\mathrm{CO}$ emission in its close vicinity: a knot about $7^{\prime \prime}(0.09 \mathrm{pc})$ to the northeast seen at -38 and $-36 \mathrm{~km} \mathrm{~s}^{-1}$ is most likely the redshifted counterpart of the compact feature seen at -70 to $-66 \mathrm{~km} \mathrm{~s}^{-1}$; a southwestern clump identifiable in the integrated map and channel maps of $\geq-48 \mathrm{~km} \mathrm{~s}^{-1}$ is probably associated with MM7 as well.

Figures 4 and 5 show the velocity integrated and channel maps, respectively, of the ${ }^{13} \mathrm{CO}(2-1)$ emission, obtained from the combined SMA and CSO data. In general the ${ }^{13} \mathrm{CO}$ emission shows a morphology similar to that of the $\mathrm{CO}$ emission, but within a narrower velocity range. The MM1 NW-SE outflow appears more predominant in the ${ }^{13} \mathrm{CO}$ emission. Although fainter, the $\mathrm{PA} \sim 40^{\circ}$ and $0^{\circ}$ branches of the blueshifed emission are both identifiable. The redshifted emission of the MM7 outflow is clearly seen; in particular in the channel map of $-48 \mathrm{~km} \mathrm{~s}^{-1}$ it shows a bi-conical structure.

A schematic view of the multiple outflows detected here is shown in Figure 6, which presents a tentative anatomy of the complicated outflow structures seen in $\mathrm{CO}$ and ${ }^{13} \mathrm{CO}$ $(2-1)$. A more detailed discussion on the MM1 outflow is presented in Section 4.2.

Following Qiu et al. (2009), the outflow parameters, including the mass $\left(M_{\text {out }}\right)$, mo- 
mentum $\left(P_{\text {out }}\right)$, energy $\left(E_{\text {out }}\right)$, dynamical timescale $\left(t_{\text {dyn }}\right)$, mass outflow rate $\left(\dot{M}_{\text {out }}\right)$ and momentum outflow rate $\left(\dot{P}_{\text {out }}\right)$, are calculated. The optical depth effect as a function of velocity and spatial distribution is corrected by comparing the $\mathrm{CO}$ with ${ }^{13} \mathrm{CO}$ fluxes and adopting a C-to- ${ }^{13} \mathrm{C}$ ratio of 79 (Wilson \& Rood 1994). The derived parameters are listed in Table 2, To avoid contamination from ambient diffuse gas, we conservatively calculate the mass of the outflowing gas at velocities of $\leq-64$ and $\geq-48 \mathrm{~km} \mathrm{~s}^{-1}$, which are more than $6 \mathrm{~km} \mathrm{~s}^{-1}$ away from the systemic velocity of $-57.4 \mathrm{~km} \mathrm{~s}^{-1}$ (van der Tak et al. 2000). However, from Figure 3, the emission at -62 and $-50 \mathrm{~km} \mathrm{~s}^{-1}$ has significant contribution from outflows. If we choose the lowest outflow velocities to be -62 and $-50 \mathrm{~km} \mathrm{~s}^{-1}$, the gas mass amounts to $110 M_{\odot}$. Hence, the total outflow mass should be in the range of 50 to 100 $M_{\odot}$.

\subsection{High-density Tracing Molecular Lines}

\subsubsection{Emission and absorption toward MM1}

MM1 shows molecular chemistry typical of a hot molecular core (HMC, Cesaroni 2005; Beuther et al. 2009). Excluding $\mathrm{CO}$ and ${ }^{13} \mathrm{CO}(2-1)$, around 70 lines arising from 15 molecular species are detected toward MM1 with peak fluxes above $6 \sigma$ (Table 3). Spatially unresolved emission from complex O-bearing molecules, e.g., methyl formate $\left(\mathrm{CH}_{3} \mathrm{OCHO}\right)$ and ethanol $\left(\mathrm{C}_{2} \mathrm{H}_{5} \mathrm{OH}\right)$, and N-bearing molecules, e.g., methyl cyanide $\left(\mathrm{CH}_{3} \mathrm{CN}\right)$ and isocyanic acid (HNCO), is clearly seen. In particular, the number of detected lines is largely dominated by O-bearing molecules, e.g., $\mathrm{CH}_{3} \mathrm{OH}, \mathrm{CH}_{3} \mathrm{OCHO}$, and $\mathrm{C}_{2} \mathrm{H}_{5} \mathrm{OH}$.

The $\mathrm{CH}_{3} \mathrm{CN}$ emission is an excellent temperature and density probe (Arava et al. 2005; Cesaroni 2005). The $\mathrm{CH}_{3} \mathrm{CN}$ lines detected in MM1 cover a wide range of upper level energies above the ground state, $E_{\text {up }}(69-526 \mathrm{~K})$. Following Qiu \& Zhang (2009), we perform a simultaneous fitting to all the detected $K$ components of the $\mathrm{CH}_{3} \mathrm{CN}$ (12-11) emission, where the temperature, column density, and source size are determined by means of grid search $\chi$-square minimization. In Figure 7, the best fit model agrees very well with the observation, and yields a temperature of $245_{-25}^{+25} \mathrm{~K}$, a column density of $5_{-1}^{+1} \times 10^{16} \mathrm{~cm}^{-2}$, and a source size of $1450_{-130}^{+170} \mathrm{AU}$. As the fitting is highly nonlinear, uncertainties are determined from a Monte Carlo analysis accounting for the $1 \sigma$ noise and the $\sim 10 \%$ uncertainty of flux calibration. Compared to the rotation diagram method, the fitting improves the parameter derivation by solving for the optical depths (Goldsmith \& Langer 1999), which range from 0.16 for the $K=8$ line to 2.99 for the $K=3$ line in the best fit model. The fitting, however, simply assumes uniform gas. Density and temperature gradients certainly

exist in a gas and dust envelope surrounding a (proto)star (e.g., Scoville \& Kwan 1976; 
Osorio et al. 2009). The best fit model thus represents a characteristic estimate. The beamaveraged $\mathrm{CH}_{3} \mathrm{CN}$ column density, $3 \times 10^{15} \mathrm{~cm}^{-2}$, can be compared to the $\mathrm{H}_{2}$ column density $\left(1.4 \times 10^{24} \mathrm{~cm}^{-2}\right.$, derived from the dust continuum peak) for an estimate of the $\mathrm{CH}_{3} \mathrm{CN}$ abundance, which is $2.1 \times 10^{-9}$, in agreement with typical values of $10^{-10^{-}}-10^{-8}$ measured in other HMCs (e.g., Hatchell et al. 1998; Chen et al. 2006; Zhang et al. 2007). Finally, the $\mathrm{C}_{2} \mathrm{H}_{5} \mathrm{OH}\left(13_{1,13}-12_{0,12}\right)\left(\nu_{0}=220601.93 \mathrm{MHz}\right)$ line in Figure 7 is often identified as $\mathrm{CH}_{3}{ }^{13} \mathrm{CN}$ $\left(12_{3}-11_{3}\right) \quad\left(\nu_{0}=220599.98 \mathrm{MHz}\right)$ in the literature, which would imply a $\mathrm{CH}_{3} \mathrm{CN}\left(12_{3}-11_{3}\right)$ optical depth of $\sim 42$, abnormally high for such a high-density and warm gas tracer. If the optical depths were that high the low $K$ components of the $\mathrm{CH}_{3} \mathrm{CN}$ (12-11) emission would be entirely optically thick and show flattened line peaks; this clearly disagrees with the observed spectra. Here we believe the emission arising from $\mathrm{C}_{2} \mathrm{H}_{5} \mathrm{OH}\left(13_{1,13}-12_{0,12}\right)$.

A secondary emission feature around $-50 \mathrm{~km} \mathrm{~s}^{-1}$ is seen in Nitrogen- and Sulfur-bearing species, i.e., $\mathrm{CH}_{3} \mathrm{CN}$, HNCO, OCS, $\mathrm{SO}$, and $\mathrm{SO}_{2}$. The feature is not detected $(<6 \sigma)$ in lines of $\mathrm{H}_{2}^{13} \mathrm{CO}, \mathrm{CH}_{3} \mathrm{OH}$, or any other large O-bearing molecules. The emission is probably arising from an interaction between the outflowing material and a clump residing in the far side of the central young O star. The lines in Nitrogen- and Sulfur-bearing species could be related to shock-induced chemistry, while the deficiency in lines of $\mathrm{CH}_{3} \mathrm{OH}, \mathrm{H}_{2}^{13} \mathrm{CO}$, and larger Obearing molecules could be due to an initially low abundance of the "key" species $\mathrm{CH}_{3} \mathrm{OH}$ and $\mathrm{H}_{2} \mathrm{CO}$ for the clump (e.g., Charnley et al. 1992). The existing observations cannot shed further light on this issue.

Thought to be a probe of inward motion of dense gas surrounding a forming star, molecular lines with redshifted absorption observed at high-angular-resolutions have been reported in a very few high-mass star-forming cores (e.g., Zhang et al. 1997; Beltrán et al. 2006). Toward MM1, an inverse P-Cygni profile with redshifted absorption $(<-6 \sigma)$ is seen in lines of $\mathrm{C}^{18} \mathrm{O}(2-1)$, SO $\left(5_{6}-4_{5}\right)$, $\mathrm{HNCO}\left(10_{0,10}-9_{0,9}\right), \mathrm{CH}_{3} \mathrm{OH}\left(8_{-1,8}-7_{0}, 7\right) E$ and $\left(8_{0,8}-7_{1}, 6\right) E$, and $\mathrm{CH}_{3} \mathrm{CN}$ (12-11) $K=2,3$ (see, e.g., Figure 8). In these lines, the absorption comes from dense gas lying on the near side of the central continuum source, absorbing bright continuum emission and moving toward the source (away from the observer). Meanwhile, the emission is attributed to dense gas residing on the far side of the central source and moving toward the observer; within a $3^{\prime \prime} \times 2^{\prime \prime}$ beam, dense gas with velocities largely along the plane of sky (i.e., orthogonal to the line of sight) can contribute to the emission as well. The lines showing redshifted absorption have $E_{\text {up }}$ spanning a range of $16-133 \mathrm{~K}$. Roughly speaking, the absorption in lines of higher excitation (e.g., the $\mathrm{CH}_{3} \mathrm{OH}$ and $\mathrm{CH}_{3} \mathrm{CN}$ lines) may trace inner, warmer gas with velocities mostly along the line of sight, hence appears more biased toward redshifted velocities. In addition, analytical models of a dense core under self-similar gravitational collapse shows that the infall velocity scales with the radius as $r^{-0.5}$ (Larson 1972; Shu 1977), which may partly contribute to the redward shift of the absorption 
in lines of higher $E_{\mathrm{up}}$. Assuming the collapse proceeds in a spherical geometry, the accretion rate can be estimated following $\dot{M}_{a c c}=4 \pi r^{2} \rho V_{\text {infall }}$, where $\dot{M}_{a c c}$ is the mass accretion rate, $r$ the radius of the infalling envelope, $\rho$ the mass density, and $V_{\text {infall }}$ the infall velocity. From the dust continuum peak the $\mathrm{H}_{2}$ number density is about $10^{7} \mathrm{~cm}^{-3}$ averaged over $r \sim 3000$ AU. The measured infall velocity is about $2.5 \mathrm{~km} \mathrm{~s}^{-1}$. As an order-of-magnitude estimate, $\dot{M}_{a c c}$ amounts to $3 \times 10^{-3} M_{\odot} \mathrm{yr}^{-1}$. However, the accretion may considerably deviate from a spherical geometry, in particular for the inner part closely surrounding the central source. The free-free emission in IRS 1 shows a double-lobed morphology at the center, suggesting that the accretion proceeds along a flattened structure perpendicular to the ionized gas elongation. If the collapse occurs inside a solid angle $\Omega, \dot{M}_{a c c}$ scales to $\Omega /(4 \pi) \cdot 3 \times 10^{-3}$ $M_{\odot} \mathrm{yr}^{-1}$.

\subsubsection{Emission from new cores MM2 to MM9}

In addition to MM1, the dust continuum map reveals eight new cores, MM2-9. The $\mathrm{C}^{18} \mathrm{O}(2-1)$, SO $\left(5_{6}-4_{5}\right)$, and $\mathrm{CH}_{3} \mathrm{OH}\left(8_{-1,8}-7_{0,7}\right) E$ lines show emission from part of these new cores and extended structures, and are not significantly affected by outflows or ambient diffuse gas. Figure 9 shows moment-0 (velocity averaged) maps of these lines.

The $\mathrm{C}^{18} \mathrm{O}(2-1)$ emission is dominated by two dense clumps residing on both sides of an absorption feature around MM1: the western clump peaks at MM2 and shows extension from MM5 to MM4; the eastern clump encompasses MM3 and MM6. While MM2 coincides

with the brightest $\mathrm{C}^{18} \mathrm{O}$ peak, cores MM3-6 are more or less offset from local $\mathrm{C}^{18} \mathrm{O}$ peaks. Another interesting feature in $\mathrm{C}^{18} \mathrm{O}$ is a $\mathrm{C}$-shaped shell seen in the southeast of the $\mathrm{mm}$ cores. In comparison with the $\mathrm{CO}$ and ${ }^{13} \mathrm{CO}(2-1)$ emission, the $\mathrm{C}^{18} \mathrm{O}$ shell lies immediately ahead of the brightest and furthest $\mathrm{CO} /{ }^{13} \mathrm{CO}$ clump in the redshifted outflow (see Figure 2 and Figure 4). This suggests that the C-shaped structure forms from ambient gas which is compressed by the outflow from MM1.

Apart from emission and absorption toward MM1, the SO $\left(5_{6}-4_{5}\right)$ map shows bright emission around MM2, MM3, MM6, MM8, MM9, as well as faint extensions toward MM4 and MM5. The SO map also shows clumps without a counterpart in the dust continuum; for example, two bright clumps to the southeast of MM6 are not seen in dust continuum or any other line. It is unclear whether the SO emission in the two clumps is associated with the MM6 outflow or arising from protostellar cores whose dust continuum is below the detection limit of our SMA observation.

Compared to $\mathrm{C}^{18} \mathrm{O}(2-1)$ and $\mathrm{SO}\left(5_{6}-4_{5}\right)$, the $\mathrm{CH}_{3} \mathrm{OH}\left(8_{-1,8}-7_{0,7}\right) E$ line has a consider- 
ably higher $E_{\text {up }}(89 \mathrm{~K})$ and appears to trace more compact structures. In addition to MM1, cores MM3, MM6, MM7, MM8, and MM9 are clearly detected in this line. The $\mathrm{CH}_{3} \mathrm{OH}$ peaks approximately coincide with the dust continuum peaks, indicating the presence of internal heating within each of the detected cores. Relatively faint emission associated with MM5 is detected as well.

A serendipitous discovery from the $\mathrm{CH}_{3} \mathrm{OH}\left(8_{-1,8}-7_{0,7}\right) E$ map is a new $229.8 \mathrm{GHz}$ maser at (R.A., decl. $)_{\mathrm{J} 2000}=\left(23^{\mathrm{h}} 13^{\mathrm{m}} 45.552,61^{\circ} 27^{\prime} 411^{\prime \prime} 5\right)$, which is seen as unresolved and bright emission with a peak flux of $\sim 1.64 \mathrm{Jy}_{\text {beam }}{ }^{-1}$ and a narrow line width of less than 2 $\mathrm{km} \mathrm{s}^{-1}$ (Figure 9k and Figure 10). It is difficult to assess whether this maser spot is associated with any of the mm continuum sources which are more than $0.2 \mathrm{pc}$ away. Another possibility is that the maser is associated with a compact $\mathrm{CH}_{3} \mathrm{OH}\left(8_{-1,8}-7_{0,7}\right) E$ and $\mathrm{SO}\left(5_{6}-4_{5}\right)$ clump about $5^{\prime \prime}$ to the north. The clump probably traces a low- to intermediate-mass protostellar core whose $1.3 \mathrm{~mm}$ dust emission is below the detection limit of our SMA observation. More interestingly, the maser spot lies right at the tip of a compact CO outflow (Figure 2), suggesting that the masing line is pumped by the outflow shocks, which is consistent with the class I property of the transition. There has been very few report on the detection of 229.8 GHz methanol maser (Slysh et al. 2002; Qiu \& Zhang 2009), probably because of its high frequency hence not covered by previous methanol maser surveys.

In the VLA NH $3(1,1)$ and $(2,2)$ data taken by Zheng et al. (2001), emission in MM69 is clearly seen in both transitions (see Figure 11), while an investigation of emission in MM2-5 is severely affected by predominant absorption arising from MM1. As there is no clear detection of a satellite line in Figure 11, we assume the emission to be optically thin, and deduce rotational temperatures, $T_{R}$, from the intensity ratio of the two transitions following $T_{R}=-41.7 / \ln \left(0.282 T_{2,2 ; m} / T_{1,1 ; m}\right)$, where $T_{1,1 ; m}$ and $T_{2,2 ; m}$ are the brightness temperatures of the main hyperfine components of the $\mathrm{NH}_{3}(1,1)$ and $(2,2)$ transitions, respectively (Ho \& Townes 1983); the formula assumes equal line width for the two transitions, which is probably a good approximation but difficult to verify given the moderate sensitivity and spectral resolution. We obtain $T_{R}$ of $29 \pm 13,34 \pm 12,33 \pm 14$, and $27 \pm 7 \mathrm{~K}$ for MM6-9, respectively; the uncertainties account for the $1 \sigma$ noise of the spectra. Correcting for the depopulation effect (Danby et al. 1988), we estimate the kinetic temperatures from $T_{R}$ to be $43,58,54$, and $38 \mathrm{~K}$ for MM6-9, respectively. 


\section{Discussion}

\subsection{Nature of the MM1 core}

The MM1 core has a size of about $0.05 \mathrm{pc}$, an $\mathrm{H}_{2}$ number density of order $10^{7} \mathrm{~cm}^{-3}$, a characteristic temperature of $245 \mathrm{~K}$, and rich chemistry in complex organic molecules. All these indicate that MM1 is a typical HMC. On the other hand, the presence of bright IR and radio emission suggests that the core is at a relatively more evolved stage compared to many other HMCs (e.g., Chen et al. 2006; Zhang et al. 2007; Girart et al. 2009; Qiu \& Zhang 2009). The core also shows particularly rich chemistry in large O-bearing molecules (e.g., $\mathrm{CH}_{3} \mathrm{OCHO}$ and $\mathrm{C}_{2} \mathrm{H}_{5} \mathrm{OH}$ ), consistent with a relatively more evolved stage.

According to stellar structure models of Schaller et al. (1992), the central young star embedded within MM1 has a mass of $\gtrsim 25 M_{\odot}$. Redshifted absorption seen in several lines toward MM1 implies the presence of an accretion flow onto the central star or stardisk system which may further increase the mass of the young $\mathrm{O}$ star. The $\sim 20 M_{\odot}$ core, however, is not equivalent to the gas reservoir of the accretion. On the one hand, Myers (2009) found that clustered and high-density regions form high-mass protostars from both core and environment gas. There are also claims that the accretion onto a forming O star may be fed by global infall of the parent cloud (e.g., Galván-Madrid et al. 2009). For NGC 7538 IRS 1, it is unclear whether there is a global infall pertaining to the accretion. Previous $\mathrm{NH}_{3}(1,1)$, (2,2) observations revealed blueshifted absorption toward IRS 1 (Wilson et al. 1983; Henkel et al. 1984; Keto 1991, also see Figure 8), which is attributed to expanding or outflowing gas moving toward the observer. The $\mathrm{NH}_{3}$ absorption is optically thick ( $\tau \gg 1$, Henkel et al. 1984; Keto 1991), presumably tracing gas mostly residing in outer layers surrounding the HMC. Thus the bulk of the outer gas appears to be in an outward motion. On the other hand, various mechanisms, e.g., outflows, heating and ionization, and accretion by nearby protostars, can disperse dense gas and conspire to terminate

the accretion (Myers 2009). In particular, Keto (2007) described a three-stage evolutionary sequence of an Hir region around a massive young star. In his model, the dynamics and morphology of the ionized gas are determined by the ratio of the ionization radius, $R_{i}$, and the gravitational radius, $R_{b}$, defined by $G M_{*} / 2 c^{2}$, where $M_{*}$ is the mass of the star and $c$ is the sound speed. With $M_{*}=25 M_{\odot}$ and $c=10 \mathrm{~km} \mathrm{~s}^{-1}$ (for ionized gas temperature of $\left.10^{4} \mathrm{~K}\right), R_{b}$ is $110 \mathrm{AU}$. The innermost ionized gas around IRS 1 has a radius of $\sim 500$ AU (Campbell 1984; Gaume et al. 1995; Sandell et al. 2009). Thus, for IRS $1, R_{i}>R_{b}$, corresponding to the second to third stages in Keto's model, that is, the ionized gas moves outward and progressively expands toward the equatorial plane. In this picture the accretion is confined to a narrow range of angle around the disk and is close to termination. 


\subsection{The MM1/IRS 1 outflow}

Multiple outflows are seen in the $\mathrm{CO}$ and ${ }^{13} \mathrm{CO}$ maps. However, the bulk outflowing gas is centered at the brightest core MM1 which harbors the IRS 1 young O star. As outlined in Figure 6, the blueshifted lobe of the MM1 outflow is dominated by a slightly curved filament with a PA of about $-50^{\circ}$. In sub-arcsec resolution observations, an elongated feature with an orientation similar to that of the $\mathrm{CO} /{ }^{13} \mathrm{CO}$ filament is seen in radio continuum $\left(\lesssim 1^{\prime \prime}\right.$, Campbell 1984; Gaume et al. 1995; Sandell \& Sievers 2004) and mid-IR emission $\left(\sim 4^{\prime \prime}\right.$, De Buizer \& Minier 2005). The $0.5 \mathrm{pc}$ structure seen in $\mathrm{CO}$ and ${ }^{13} \mathrm{CO}(2-1)$ can be traced back to about $0 . / 2(\sim 500 \mathrm{AU})$ from the central source; within $r \sim 0 . \prime 2$, the ionized gas shows a double-lobed morphology in a north-south orientation. In addition, near-IR observations toward IRS 1 reveals a fan-shaped structure (opening angle $\sim 90^{\circ}$ ) extending from IRS 1 to the northwest $\left(\sim 10^{\prime \prime}\right.$, Kraus et al. 2006). On a larger scale, the $\mathrm{CO} /{ }^{13} \mathrm{CO}$ filament is seen projected against a dense clump revealed by the $\mathrm{NH}_{3}$ emission (see Figure 6). The most likely scenario capable of incorporating the inner ionized gas with a north-south orientation and the northwestern structure extending from 500 AU to 0.5 pc from the central source seems to be a wide-angle wind from IRS 1 which carves an outflow cavity. The axis of the wide-angle wind is mostly along the north-south orientation and slightly inclined to the west. The northwestern structure observed in radio, mid-IR, and in $\mathrm{CO}$ and ${ }^{13} \mathrm{CO}(2-1)$ emission traces the western wall of the outflow cavity. The lack of appreciable emission from the eastern wall is likely ascribed to the deficiency of dense gas to the northeast of IRS 1 (e.g., Zheng et al. 2001). Indeed, from a close inspection of the ionized gas and hot dust immediately around IRS 1, faint extension to the northeast does exist as well (Figure 2 in Campbell 1984; Figure 1b in Gaume et al. 1995; Figure 2b in De Buizer \& Minier 2005). The CO $/{ }^{13} \mathrm{CO}$ structure originating from the vicinity of IRS 3 and MM4 and extending to the north $\left(\mathrm{PA} \sim 0^{\circ}\right)$ is puzzling; in Figure 2 and Figure 4, the structure seems to be part of the MM1 outflow but with an orientation very different from the inner part (i.e., the PA of the outflow varying from $-50^{\circ}$ to $0^{\circ}$ ). In dust continuum or $\mathrm{NH}_{3}$ maps of the region (Zheng et al. 2001; Sandell \& Sievers 2004; Reid \& Wilson 2005; Pestalozzi et al. 2006), there appears to be no dense gas immediately to the west of the structure, excluding the possibility of being confined by ambient dense gas. From Figure 6 , the wide-angle wind seems to be expanding into an ambient dense clump seen in $\mathrm{NH}_{3}$ and

as a consequence of this interaction, part of the gas is deflected to the north, forming the $\mathrm{PA} \sim 0^{\circ}$ structure. However, with the existing data it cannot be ruled out that the structure is associated with IRS 3 or MM4.

It is not straightforward either to unambiguously identify and interpret the redshifted lobe of the MM1 outflow. In the wide-angle wind scenario proposed above, the structure mostly consists of ambient gas being entrained or swept up by the wide-angle wind and 
tracing the eastern wall of the southern outflow cavity. The slightly distorted appearance of the structure could be partly caused by interaction with other outflows (e.g., the MM7 outflow). An interesting feature is the $\mathrm{C}$-shaped shell seen in $\mathrm{C}^{18} \mathrm{O}$. It lies ahead of the furthest and brightest $\mathrm{CO} /{ }^{13} \mathrm{CO}$ clump and features dense gas being compressed by part of the leading front of the wide-angle wind. It is puzzling that the western wall of the southern outflow cavity is unseen in $\mathrm{CO}$ or ${ }^{13} \mathrm{CO}$, as at least in projection dense gas to the southwest of MM1 is seen in $\mathrm{NH}_{3}$ and dust continuum. One possibility is that in a three-dimensional picture the southern lobe of the wide-angle wind is expanding into a medium without dense gas in the west and cannot produce appreciable emission.

Hollenbach et al. (1994) studied the photoevaporation of disks around massive young stars and developed a model of the photoevaporative wind from the disk. Lugo et al. (2004) performed a parametric investigation of such a wind in NGC 7538 IRS 1 by modeling the freefree emission as arising from the wind and found a mass-loss rate of $1.1 \times 10^{-5} M_{\odot} \mathrm{yr}^{-1}$. As the speed of the photoevaporative wind is $\sim 10-50 \mathrm{~km} \mathrm{~s}^{-1}$ (Hollenbach et al. 1994), comparable to that of CO outflows, the mass loss rate of the wind seems too low to drive the NGC 7538 IRS 1 outflow, whose mass loss rate is of order $10^{-3} M_{\odot} \mathrm{yr}^{-1}$ (Table 2). The photoevaporative wind may play a role in driving the IRS 1 molecular outflow, however, the large amount of gas mass, momentum, and energy in outflowing gas requires a more energetic wind as its driving engine. In a momentum-driven picture (working like a snow-plow), a fast, 1000 $\mathrm{kms}^{-1}$ wind with a mass loss rate of $10^{-5} M_{\odot} \mathrm{yr}^{-1}$ is able to drive the observed molecular outflow; in an energy-driven situation (working by ram pressure), the required mass loss rate is much lower, i.e., $10^{-7} M_{\odot} \mathrm{yr}^{-1}$, provided the wind speed reaches $1000 \mathrm{~km} \mathrm{~s}^{-1}$. Stellar winds from $\mathrm{O}$ stars and possible accretion-driven winds (e.g., a high-mass analogue of $X$ winds in low-mass protostars) are expected to have velocities reaching $\sim 1000 \mathrm{~km} \mathrm{~s}^{-1}$, and energy-wise, are likely to drive the massive and energetic molecular outflow.

\subsection{A massive cluster in the making}

In single-dish submm and mm continuum maps of the NGC 7538 complex, the most remarkable cloud has a size of about 1 pc and slightly extends to the southeast (Sandell \& Sievers 2004; Reid \& Wilson 2005; Pestalozzi et al. 2006). Star formation activity within this cloud has long been thought to be concentrated around IRS 1-3, and IRS 1 is the source that attracted most of the interest. Our SMA $1.3 \mathrm{~mm}$ continuum observation uncovers a total of nine cores with a spatial distribution roughly following the shape of the cloud. Apart from MM1, which harbors the IRS 1 young O star, cores MM2-9 are new detections. The detection of these dusty cores is interesting in the context of cloud fragmentation and cluster formation. 
For MM1, with a number density of $1.7 \times 10^{7} \mathrm{~cm}^{-3}$ (averaged within a beam and assuming a spherical geometry) and a temperature of $245 \mathrm{~K}$, one derives a thermal Jeans mass $\left(M_{J}\right)$ of $17 M_{\odot}$, which is comparable to the measured core mass and compatible with the scenario that heating from the forming star may have helped to suppress fragmentation of the envelope (e.g., Krumholz 2006; Krumholz \& McKee 2008). However, all the new cores are very young, apparently lacking significant heating. These cores are not detected in $\mathrm{CH}_{3} \mathrm{CN}$, and only part of them are seen in one transition of $\mathrm{CH}_{3} \mathrm{OH}$ (see Figure 9). For MM6-9, the $\mathrm{NH}_{3}$ data reveal temperatures of order $50 \mathrm{~K}$ (Section 3.3.2). For MM2-5, a rough upper limit may be obtained based on the absence of $\mathrm{CH}_{3} \mathrm{CN}$ emission, which is a typical thermometer of HMCs. For example, cores MM2-5 have masses close to MM1; if the abundance ratio and spatial distribution of $\mathrm{CH}_{3} \mathrm{CN}$ in $\mathrm{MM} 2-5$ resembles that in MM1, at a temperature of $100 \mathrm{~K}$, the brightness temperature (averaged within a beam of $3^{\prime \prime} \times 2^{\prime \prime}$ ) of the $\mathrm{CH}_{3} \mathrm{CN}(12-11) \mathrm{K}=0-3$ emission in MM2-5 would be about $2-4 \mathrm{~K}$, which could have been detected at a $\gtrsim 15 \sigma$ level. Alternatively, with the $\mathrm{CH}_{3} \mathrm{CN}$ (12-11) $K$-ladder observed with the SMA, Qiu \& Zhang (2009) derived a temperature of $110 \mathrm{~K}$ for a HMC in the HH 80-81 massive star-forming region; if the $\mathrm{HH} 80-81 \mathrm{HMC}$ were at a distance of $2.65 \mathrm{kpc}$ and observed with a $3^{\prime \prime} \times 2^{\prime \prime}$ beam, the brightness temperature of the $K=0,1$ components would have been around $1 \mathrm{~K}$; cores MM2-5 in NGC 7538 have masses comparable to that of the $\mathrm{HH} 80-81 \mathrm{HMC}$, while the $\mathrm{CH}_{3} \mathrm{CN}$ emission is not detected $(\sigma \sim 0.13 \mathrm{~K})$. All this indicates that MM2-5 have temperatures well below 100 K. Sandell \& Sievers (2004) found a dust temperature of $40 \mathrm{~K}$ for submm condensations around IRS 1 . This probably provides a rough estimate of the averaged temperature of MM2-9, as part of them roughly coincide with the submm condensations.

MM2-9 have number densities of $\sim 0.4-1.2 \times 10^{7} \mathrm{~cm}^{-3}$, or mass column densities $\gtrsim 1$ $\mathrm{g} \mathrm{cm}^{-2}$, matching a proposed threshold of $1 \mathrm{~g} \mathrm{~cm}^{-2}$ for molecular clouds capable of avoiding fragmentation and forming massive stars (Krumholz \& McKee 2008). But the masses of the new cores are well above $M_{J}$, implying the importance of supersonic turbulence and/or magnetic field in cloud fragmentation and possible star formation within the new cores (e.g., Zhang et al. 2009). While an investigation of the magnetic field awaits new observations, a glimpse of turbulence can be obtained by looking into the emission lines arising from these cores. Figure 12 shows spectra in $\mathrm{C}^{18} \mathrm{O}(2-1)$, $\mathrm{SO}\left(5_{6}-4_{5}\right)$, and $\mathrm{CH}_{3} \mathrm{OH}\left(8_{-1,8^{-}} 7_{0,7}\right) E$ at the peaks of MM2-9. A gaussian fitting is performed on lines with sufficient signal-to-noise ratios. The derived line widths of $\sim 2.5-4 \mathrm{~km} \mathrm{~s}^{-1}$ are more than 5 times larger than the thermal line width. This strongly suggests the presence of supersonic turbulence which may play an important role in suppressing fragmentation, if the line widths are not dominated by kinematics related to star formation processes (e.g., outflow, infall, and rotation).

Information on potential ongoing star formation within the new cores can be obtained 
from their associations with $\mathrm{CO}$ outflows, $\mathrm{H}_{2} \mathrm{O}$ masers, and warm and dense gas condensations. Cores MM2, MM3, MM4, and MM7 are all associated with $\mathrm{H}_{2} \mathrm{O}$ maser emission (Figure 1), indicative of active star formation. Cores MM2, MM6, MM7, MM8, and probably MM4 as well, are associated with CO outflows (Figure 6] and Section 3.2), implying the presence of protostars within these cores. MM9 shows the faintest dust continuum; although lacking apparent association with an outflow or $\mathrm{H}_{2} \mathrm{O}$ maser spot, it is very likely internally heated by an intermediate-mass protostar as it shows centrally peaked emission in $\mathrm{CH}_{3} \mathrm{OH}$ and $\mathrm{SO}$ (Figure 9). There is no clear signature of star formation toward MM5, which also shows a relatively flattened profile in dust emission. It is more likely a gas and dust clump externally heated by IRS 2 (probably through a stellar-wind bow shock, Bloomer et al. 1998), rather than an internally heated star-forming core. In short, seven out of the eight new cores appear to be forming stars. The star-forming cores have masses of $\sim 5-24 M_{\odot}$ and high densities, and most of them show bright, centrally peaked $\mathrm{CH}_{3} \mathrm{OH}$ emission. Although $\mathrm{CH}_{3} \mathrm{OH}$ lines are seen in hot corinos (warm and dense envelopes around low-mass protostars, Ceccarelli et al. 2007), the emission could not be detected if the hot corinos were at distances $>2 \mathrm{kpc}$ as compared to the case of IRAS 16293-2422 (van Dishoeck et al. 1995; Kuan et al. 2004; Chandler et al. 2005). Thus, the new cores seen in $\mathrm{CH}_{3} \mathrm{OH}$ are expected to be forming intermediate- to high-mass stars. MM4 is not detected in $\mathrm{CH}_{3} \mathrm{OH}$ and its immediate northwest is deficient of emission in $\mathrm{C}^{18} \mathrm{O}$ and $\mathrm{SO}$; molecular line emission from this core is probably hampered by UV radiation from IRS 3, which has begun to ionize the surrounding and is not heavily obscured by a dusty cocoon. MM2 is the most massive of the new cores and coincides with the brightest $\mathrm{C}^{18} \mathrm{O}$ peak, but is not seen in $\mathrm{CH}_{3} \mathrm{OH}$. With a relatively low $E_{\text {up }}(16 \mathrm{~K})$, the $\mathrm{C}^{18} \mathrm{O}(2-1)$ emission preferentially traces column density enhancements rather than temperature increase (e.g., Wyrowski et al. 1999). The $\mathrm{CH}_{3} \mathrm{OH}\left(8_{-1,8}-7_{0}, 7\right) E$ emission (with a much higher $E_{\text {up }}$ of 89 $\mathrm{K})$ is more sensitive to the heating. Therefore, MM2 is presumably the youngest of the new star-forming cores.

The NGC 7538 molecular complex has been thought to exhibit sequential massive star formation propagating from northwest to southeast (Elmegreen \& Lada 1977; Werner et al. 1979). In this picture, the IRS 1-3 region lies ahead of the ionization front of the optical Hir region and represents an intermediate evolutionary phase between the ionizing stars of the His region in the northwest and deeply embedded massive protostars in the southeast (i.e., NGC 7538 S and IRS 9, Werner et al. 1979). By zooming in the parent cloud of IRS 1-3, our SMA observations provide new insights into this scenario. With the newly discovered star-forming cores, the IRS 1 young $\mathrm{O}$ star is not only accompanied by more evolved sources IRS 2 and IRS 3, but also surrounded by a group of intermediate- to high-mass protostars distributed over the parent cloud. There is no pronounced propagation of star formation 
across the cloud. In comparison with massive protostars in IRS 9 or NGC 7538 S, IRS 1-3 are certainly more evolved, but all the newly discovered star-forming cores are at very young evolutionary stages. In particular MM2, which is the closest to MM1, is apparently younger than IRS 9, which is visible in the near- to mid-IR (Werner et al. 1979), and even younger than NGC 7538 S, which shows bright $\mathrm{CH}_{3} \mathrm{CN}$ emission (Sandell \& Wright 2010). The pcsized cloud appears to be in an active phase of cluster formation, with around 10 OB-type stars and probably orders of magnitude more low-mass stars according to a typical IMF (initial mass function, e.g., Salpeter 1955; Kroupa 2002). Indeed, within the volume occupied by high- and intermediate-mass protostars described above, Kraus et al. (2006) in their 2.2 $\mu \mathrm{m}$ speckle images found a number of weak, compact sources. It is interesting to speculate that these are already more evolved lower mass members of the same forming star cluster of which IRS 1-3 and the new protostars (MM2-4 and MM6-9) define the higher mass end of the mass distribution. Here, a comparison with the well-studied Orion Nebular Cluster (ONC) is instructive. As argued by Palla \& Stahler (1999) and Huff \& Stahler (2006), the highest mass ONC member stars comprising the "Trapezium" at the center of the cluster are $\sim 10^{5}$ yr old, while the bulk of the lower mass cluster members formed 1-3 million years ago. In fact, one may consider IRS 1-3, MM2-4 and MM6 to be a Trapezium system in formation. The maximal physical separation between these objects, $0.30 \mathrm{pc}$, is much larger than the maximal separation between members of the ONC Trapezium, 0.047 pc, i.e., between $\theta^{1}$ Ori D and $\theta^{1}$ Ori E, assuming a distance of $417 \mathrm{pc}$ to the ONC (Menten et al. 2007). It is, however, well within the range of other Trapezium-type systems. A Trapezium-type system, as defined by Ambartsumian (1954; 1955; 1958), of which $\theta^{1}$ Ori is the prototype, is a multiple star whose separations are all roughly of the same order of magnitude. While their cosmogonic role is somewhat unclear, it is a well-established fact that such systems are frequently found as the highest mass systems at the centers of clusters of lower mass stars (Sharpless 1954). A catalog of 87 Trapezia with maximum separations, $r_{\max }$, within 0.2 pc was published by Salukvadze (1978), while Salukvadze \& Dzhavakhishvili (1988) reported 15 wider systems with $r_{\max }$ between 1.1 and $5.7 \mathrm{pc}$. Thus the dimensions of the nascent NGC 7538 IRS 1 Trapezium are well comparable to values generally found for such systems.

\section{Summary}

We present a $2-3^{\prime \prime}$ resolution study in the $1.3 \mathrm{~mm}$ waveband of the well-known massive star-forming region NGC 7538 IRS 1-3. The gas and dust core MM1, which harbors the IRS 1 young $\mathrm{O}$ star, is massive $\left(\sim 20 M_{\odot}\right)$, hot $(\sim 245 \mathrm{~K})$, and shows bright line emission in complex organic molecules. Redshifted absorption seen in several high-density tracing lines indicates that the central young O star or star-disk system is still accreting gas from MM1. 
In comparison with a theoretical model on early evolution of HII regions (Keto 2007), the accretion seems to be confined to a narrow range of angle about the equatorial plane.

Neither of the two nearby sources IRS 2 and IRS 3 is associated with a dense dusty envelope, confirming that the two luminous sources are at most moderately obscured.

Sensitive and high-angular-resolution observations also reveal eight new cores MM2-9 embedded within the parent cloud of IRS 1-3. These cores have masses much larger than the thermal Jeans mass, indicating the importance of turbulence and/or magnetic field in cloud fragmentation. The cores are seen in one or more of the $\mathrm{C}^{18} \mathrm{O}(2-1)$, SO $\left(5_{6}-4_{5}\right)$, and $\mathrm{CH}_{3} \mathrm{OH}\left(8_{-1,8}-7_{0,7}\right) E$ lines; the line widths are significantly larger than the thermal line width, attributed to supersonic turbulence and/or kinematics related to ongoing star formation. Seven out of the eight cores appear to be forming intermediate- to high-mass stars, of which the most massive ones appear to be members of a nascent Trapezium system. Characterized by the deeply embedded young O star IRS 1, two moderately obscured sources IRS 2 and IRS 3, and seven new intermediate- to high-mass protostellar cores, and taking into account presumably more unseen low-mass (proto)stars, a massive cluster is forming from a pc-sized cloud exposed to the ionization front of an optical HII region.

The $\mathrm{CO}$ and ${ }^{13} \mathrm{CO}$ maps obtained from the combined SMA and single-dish data reveals an outflow scenario far more complicated than a bipolar outflow seen in single-dish observations. Multiple outflows are seen, and the MM1/IRS 1 outflow is dominating the mass of $50 M_{\odot}$, momentum of $4.6 \times 10^{2} M_{\odot} \mathrm{km} \mathrm{s}^{-1}$, and energy of $4.9 \times 10^{46} \mathrm{erg}$ calculated for the gas moving at velocities of $\leq-64 \mathrm{~km} \mathrm{~s}^{-1}$ and $\geq-48 \mathrm{~km} \mathrm{~s}^{-1}$. Comparing our $\mathrm{CO},{ }^{13} \mathrm{CO}$ maps with the existing radio and IR observations we suggest that the MM1/IRS 1 molecular outflow is likely driven by a wide-angle wind from the forming $\mathrm{O}$ star or star-disk system, and the wind is expanding into a medium with inhomogeneous density distribution, creating asymmetric and curved structures seen in $\mathrm{CO} /{ }^{13} \mathrm{CO}$.

We are grateful to Dr. Jingwen Wu and Dr. Ruisheng Peng for help in obtaining the ${ }^{13} \mathrm{CO}(2-1)$ observations at the CSO. We acknowledge the anonymous referee for construtive comments which improved the paper. 


\section{REFERENCES}

Ambartsumian, V. A. 1954, Memoires of the Societe Royale des Sciences de Liege, 1, 293

Ambartsumian, V. A. 1955, The Observatory, 75, 72

Ambartsumian, V. A. 1958, Reviews of Modern Physics, 30, 944

Araya, E., Hofner, P., Kurtz, S., Bronfman, L., \& DeDeo, S. 2005, ApJS, 157, 279

Akabane, K. \& Kuno, N. 2005, A\&A, 431, 183

Beltrán, M. T., Cesaroni, R., Codella, C., Testi, L., Furuya, R. S., \& Olmi, L. 2006, Nature, 443,427

Beuther, H., Zhang, Q., Bergin, E. A., \& Sridharan, T. K. 2009, AJ, 137, 406

Bloomer, J. D. et al. 1998, ApJ, 506, 727

Campbell, B. 1984, ApJ, 282, L27

Campbell, B. \& Thompson R. I. 1984, ApJ, 279, 650

Ceccarelli, C., Caselli, P., Herbst, E., Tielens, A. G. G. M., \& Caux, E. 2007, in Protostars and Planets V, ed. V. B. Reipurth et al. (Tuscon, AZ: Univ. Arizona Press), 47

Cesaroni, R. 2005, in IAU Symp. Ser. 227, Massive Star Birth: A Crossroads of Astrophysics, ed. R. Cesaroni et al. (Cambridge: Cambridge Univ. Press), 59

Chandler, C. J., Brogan, C. L., Shirley, Y. L., \& Loinard, L. 2005, ApJ, 632, 371

Charnley, S. B., Tielens, A. G. G. M., \& Millar, T. J. 1992, ApJ, 399, L71

Chen, H.-R., Welch, W. J., Wilner, D. J., \& Sutton, E. C. 2006, ApJ, 639, 975

Danby, G., Flower, D. R., Valiron, P., Schilke, P., \& Walmsley, C. M. 1988, MNRAS, 235, 229

Davis, C. J., Moriarty-Schieven, G. Eislöffel, J., Hoare, M., \& Ray, T. P. 1998, AJ, 115, 1118

De Buizer, J. M. \& Minier, V. 2005, ApJ, 628, L151

Elmegreen, B. \& C. J. Lada, 1977, ApJ, 214, 725

Galván-Madrid, R., Keto, E., Zhang, Q., Kurtz, S., Rodríguez, L. F, \& Ho, P. T. P. 2009, ApJ, 706, 1036 
Franco-Hernández, R. \& Rodríguez, L. F. 2004, ApJ, 604, L105

Gaume, R. A., Goss, W. M., Dickel, H. R. Wilson, T. L., \& Johnston, K. J. 1995, ApJ, 438, 776

Girart, J. M., Beltrán, M. T., Zhang, Q., Rao, R., \& Estalella, R. 2009, Science, 324, 1408

Glodsmith, P. F. \& Langer, W. D. 1999, ApJ, 517, 209

Hatchell, J., Thompson, M. A., Millar, T. J., \& MacDonald, G. H. 1998, A\&AS, 133, 29

Henkel, C., Wilson, T. L., \& Johnston, K. J. 1984, ApJ, 282, L93

Hildebrand, R. H. 1983, QJRAS, 24, 267

Ho, P. T. P. \& Townes, C. H. 1983, ARA\&A, 21, 239

Hollenbach, D., Johnstone, D., Lizano, S., \& Shu, F. H. 1994, ApJ, 428, 654

Huff, E. M. \& Stahler, S. W. 2006, ApJ, 644, 355

Lugo, J., Lizano, S., \& Garay, G. 2004, ApJ, 614, 807

Kameya, O., Hasegawa, T. I., Hirano, N., Takakubo, K., \& Seki, M. 1989, ApJ, 339, 222

Kameya, O., Morita, K.-I., Kawabe, R., \& Ishiguro, M. 1990, ApJ, 355, 562

Keto, E. 1991, ApJ, 371, 163

Keto, E. 2007, ApJ, 666, 976

Keto, E., Zhang, Q., \& Kurtz, S. 2008, ApJ, 672, 423

Klaassen, P., D., Wilson, C., D., Keto, E. R., \& Zhang, Q. 2009, ApJ, 703, 1308

Kraus, S. et al. 2006, A\&A, 455, 521

Kroupa, P. 2002, Science, 295, 82

Krumholz, M. R. 2006, ApJ, 641, L45

Krumholz, M. R. \& McKee, C. F. 2008, Nature, 451, 1082

Kuan, Y.-J. et al. 2004, ApJ, 616, L27

Larson, R. B. 1972, MNRAS, 157, 121 
Martin, A. H. M. 1973, MNRAS, 163, 141

Menten, K. M., Reid, M. J., Forbrich, J., \& Brunthaler, A. 2007, A\&A, 474, 515

Moscadelli, L., Reid, M. J., Menten, K. M., Brunthaler, A., Zheng, X. W., \& Xu, Y. 2009, ApJ, 693, 406

Myers, P. C. 2009, ApJ, 706, 1341

Osorio, M., Anglada, G., Lizano, S., \& D’Alessio, P. 2009, ApJ, 694, 29

Ossenkopf, V.\& Henning, T. 1994, A\&A, 291, 943

Palla, F. \& Stahler, S. W. 1999, ApJ, 525, 772

Pestalozzi, M. R., Elitzur, M., Conway, E., \& Booth, R. S. 2004, ApJ, 603, L113

Pestalozzi, M. R., Minier, V., Motte, F., \& Conway, J. E. 2006, A\&A, 448, L57

Pratap, P., Batrla, W., \& Snyder, L. E. 1989, ApJ, 341, 832

Qiu, K. \& Zhang, Q. 2009, ApJ, 702, L66

Qiu, K., Zhang, Q., Wu, J., \& Chen, H.-R. 2009, ApJ, 696, 66

Reid, M. A. \& Wilson, C. D. 2005, ApJ, 625, 891

Salpeter, E. E. 1955, ApJ, 121, 161

Salukvadze, G. N. 1978, Astrophysics, 14, 30

Salukvadze, G. N. \& Dzhavakhishvili, G. S. 1988, Ap\&SS, 142, 79

Sandell, G., Goss, W. M., Wright, M., \& Corder, S. 2009, ApJ, L31

Sandell, G. \& Wright, M 2010, ApJ, 715, 919

Sandell, G. \& Sievers, A. 2004, ApJ, 600, 269

Schaller, G., Schaerer, D., Meynet, G., \& Maeder, A. 1992, A\&AS, 96, 269

Scoville, N. Z. \& Kwan, J. 1976, ApJ, 206, 718

Scoville, N. Z., Sargent, A. I., Sanders, D. B., Claussen, M. J., Masson, C. R., Lo, K. Y., \& Phillips, T. G. 1986, ApJ, 303, 416

Sharpless, S. 1954, ApJ, 119, 334 
Shu, F. H. 1977, ApJ, 214, 488

Slysh, V. I., Kalenskii, S. V., \& Val'Tts, I. E. 2002, Astron. Rep., 46, 49

van der Tak, F. F. S., van Dishoeck, E. F., Evans II, N. J., \& Blake, G. A. 2000, ApJ, 537, 283

van Dishoeck, E. F., Blake, G. A., Jansen, D. J., \& Groesbeck, T. D. 1995, ApJ, 447, 760

Werner, M. W., Becklin, E. E., Gatley, I., Matthews, K., Neugebauer, G., \& Wynn-Williams, C. G. 1979, MNRAS, 188, 463

Wilson, T. L., Mauersberger, R., Walmsley, C. M., \& Batrla, W. 1983, A\&A, 127, L19

Wilson, T. L. \& Rood, R. 1994, ARA\&A, 32, 191

Wynn-Williams, C. G., Becklin, E. E., \& Neugebauer, G. 1974, ApJ, 187, 473

Wyrowski, F., Schilke, P., Walmsley, M. C., \& Menten, K. M. 1999, ApJ, L43

Zheng, X.-W., Zhang, Q., Ho, P. T. P., \& Pratap, P. 2001, ApJ, 550, 301

Zhang, Q., Hunter, T. R., Beuther, H., Sridharan, T. K., Liu, S.-Y., Su, Y.-N., Chen, H.-R., \& Chen, Y. 2007, ApJ, 658, 1152

Zhang, Q. \& Ho, P. T. P. 1997, ApJ, 488, 241

Zhang, Q., Ho, P. T. P., Wright, M. C., \& Wilner, D. J. 1995, ApJ, 451, L71

Zhang, Q., Wang, Y., Pillai, T., \& Rathborne, J.2009 ApJ, 696, 268 


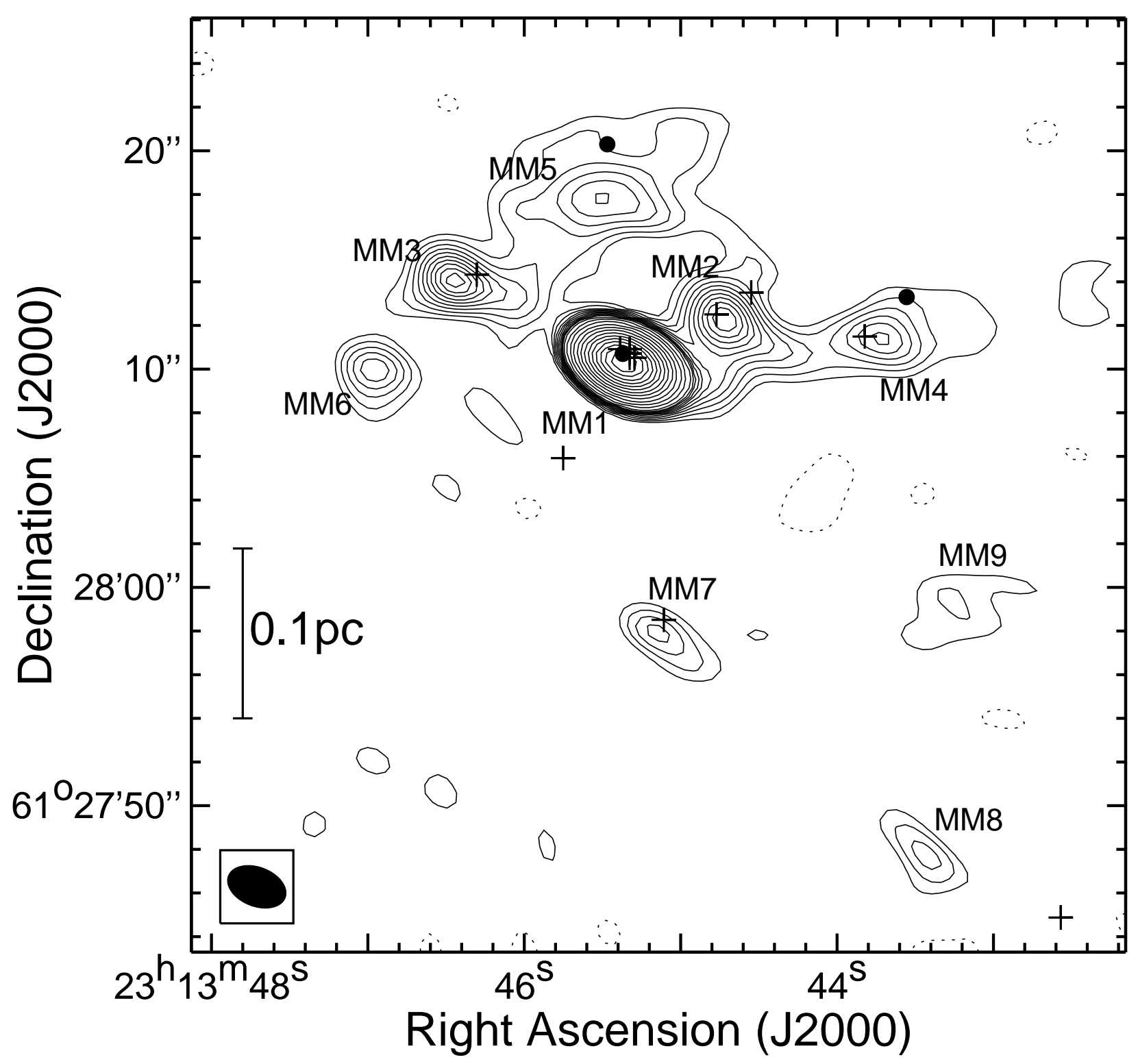

Fig. 1.- $1.3 \mathrm{~mm}$ continuum emission shown in solid contours with contour levels increasing from 30 to $165 \mathrm{mJy}_{\text {beam }}{ }^{-1}$ in steps of $15 \mathrm{mJy}_{\text {beam }}{ }^{-1}$ and continuing to $3255 \mathrm{mJy} \mathrm{beam}^{-1}$ in steps of $30 \times(1,2,3, \ldots, 12)$ mJy beam $^{-1}$; dotted contours show negative emission with absolute levels the same as that of the positive. The plus symbols depict water maser spots from Kameya et al. (1990); three filled dots mark the positions of IRS 1-3, where IRS 1 roughly coincides with MM1, IRS 2 is about $10^{\prime \prime}$ to the north and IRS 3 about $14^{\prime \prime}$ to the west. Hereafter a filled ellipse in the lower left delineates the synthesized beam at FWHM. 


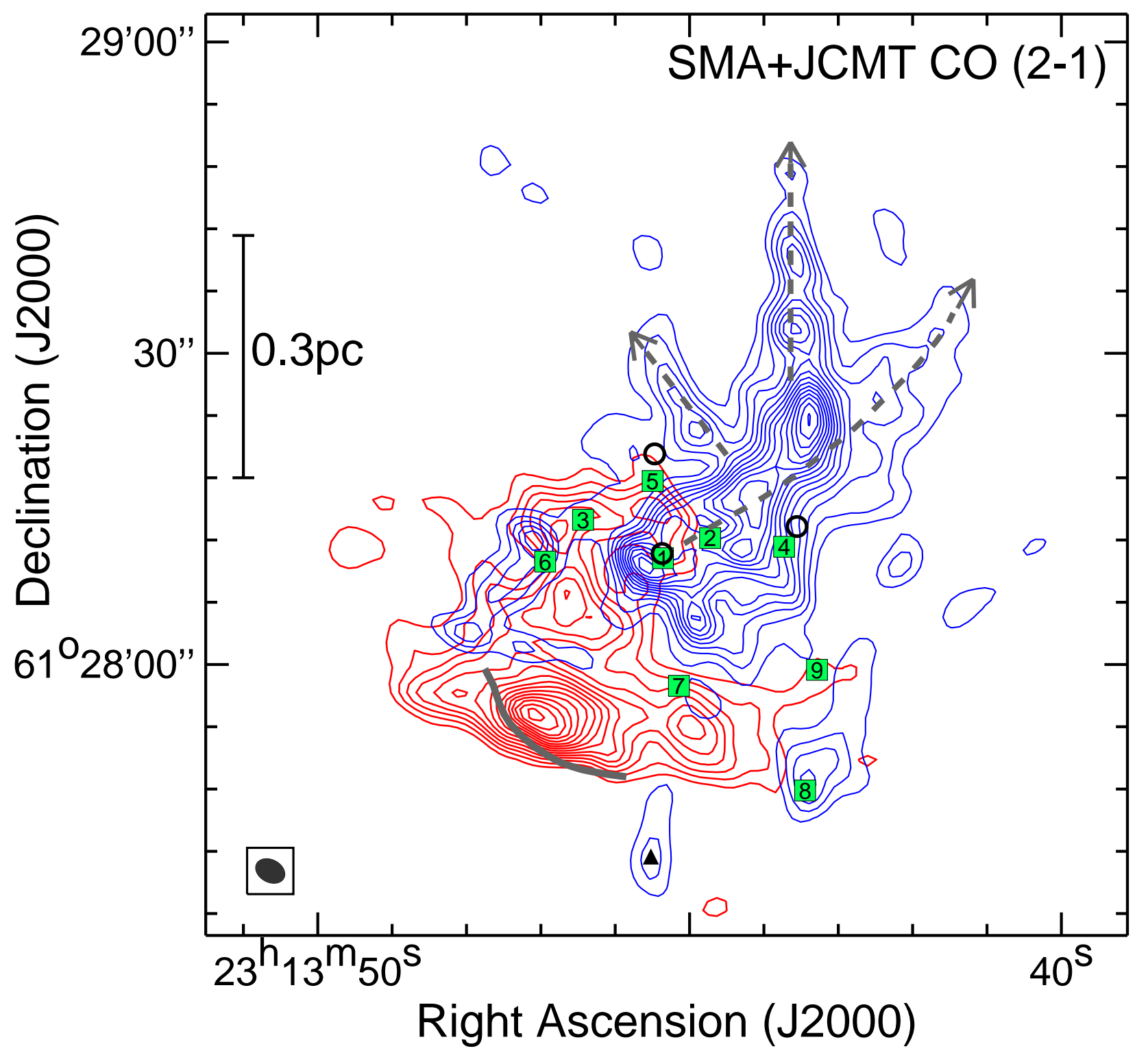

Fig. 2.- Integrated CO (2-1) emission obtained from the combined SMA and JCMT data. Blue contours show emission integrated from -78 to $-64 \mathrm{~km} \mathrm{~s}^{-1}$ and the contour levels start from $10.2 \mathrm{Jy} \cdot \mathrm{km} \mathrm{s}^{-1}$ and increase in steps of $6.8 \mathrm{Jy} \cdot \mathrm{km} \mathrm{s}^{-1}$; red contours show emission integrated from -48 to $-30 \mathrm{~km} \mathrm{~s}^{-1}$ and the contour levels start from $20.2 \mathrm{Jy} \cdot \mathrm{km} \mathrm{s}^{-1}$ and increase in steps of $10.1 \mathrm{Jy} \cdot \mathrm{km} \mathrm{s}^{-1}$. The numbered squares mark dusty cores MM1-9 (see Figure 11); three thick circles denote IRS 1-3; a filled triangle marks a newly discovered $229.8 \mathrm{GHz} \mathrm{CH}_{3} \mathrm{OH}$ maser (see Section 3.3 .2 and Figures $9 \mathrm{k}$, 10). A gray curve delineates a C-shaped structure seen in $\mathrm{C}^{18} \mathrm{O}(2-1)$ (see Section 3.3 .2 and Figure 9a), and dashed arrows outline the three "branches" of the blueshifted outflow (see Section 3.2). 


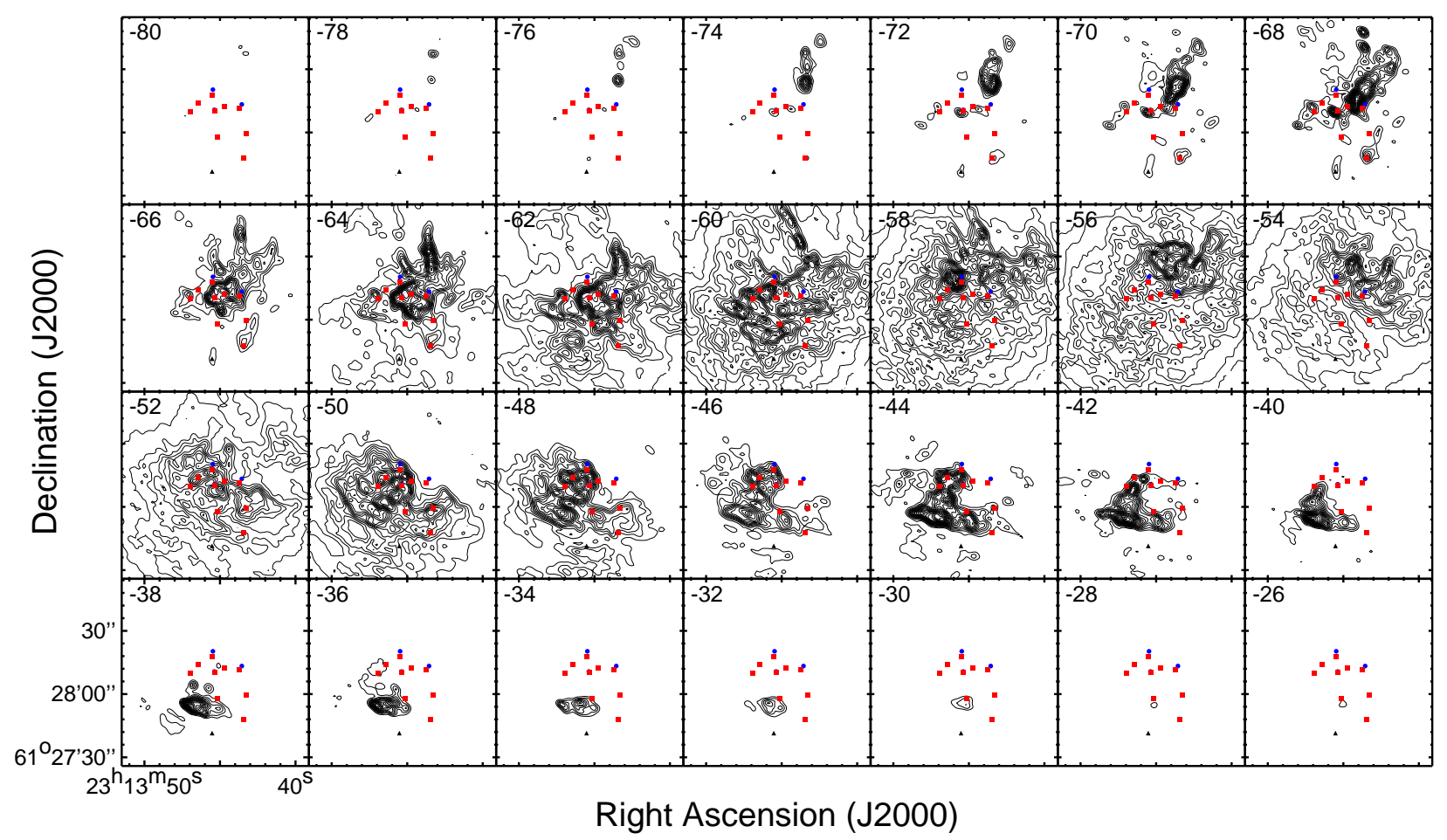

Fig. 3.- Velocity channel maps of CO (2-1) emission obtained from the combined SMA and JCMT data. The starting and spacing contour levels are $1.2 \mathrm{Jybeam}^{-1}$ for channels from -66 to $-46 \mathrm{~km} \mathrm{~s}^{-1}$ and $0.6 \mathrm{Jy}_{\text {beam }}{ }^{-1}$ in outer line wings. The central velocity of each channel is shown in the upper left of each panel; cores MM1-9 and IR sources IRS 1-3 are denoted as filled squares and dots, respectively. 


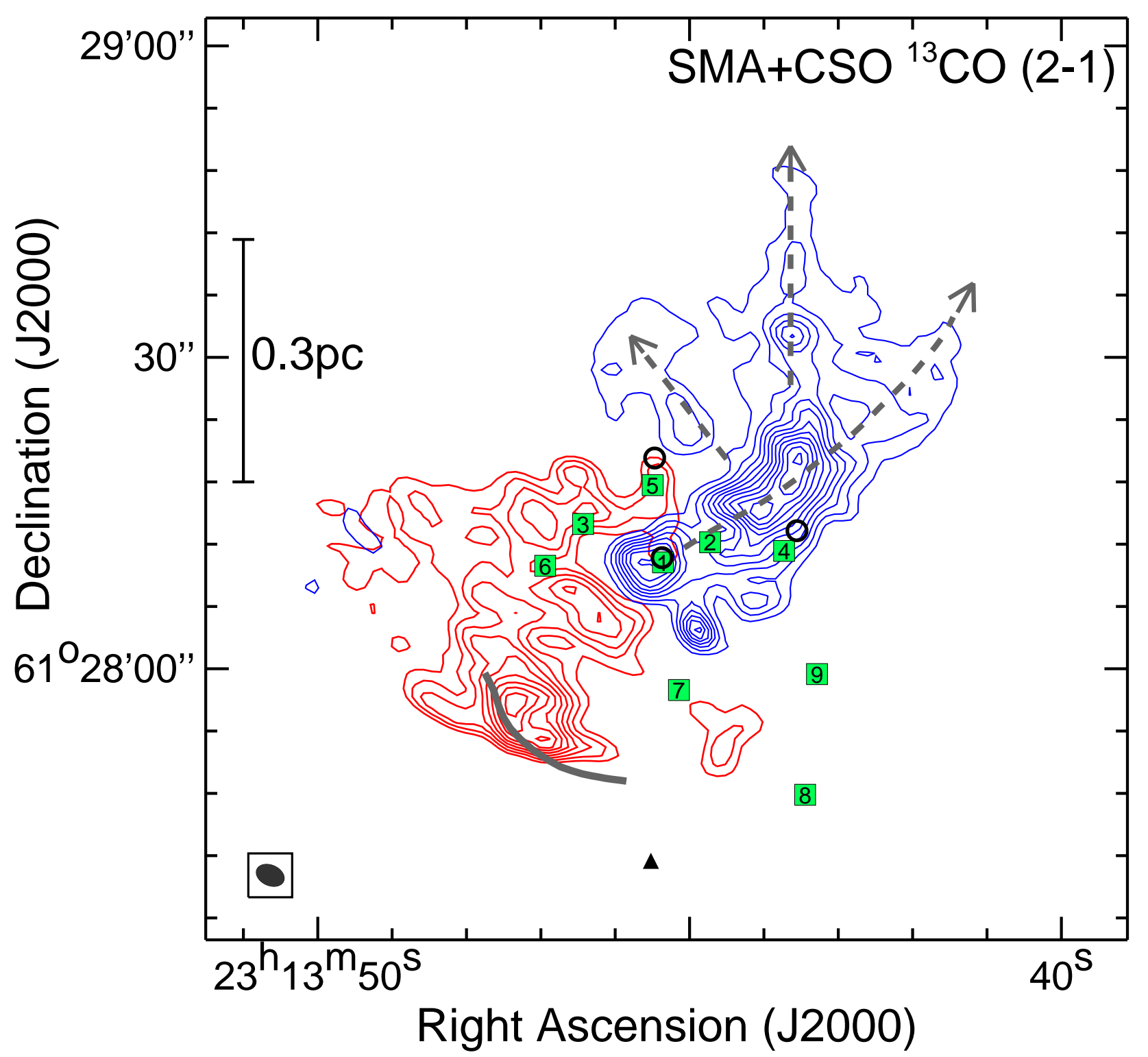

Fig. 4.- Integrated ${ }^{13} \mathrm{CO}(2-1)$ emission obtained from the combined SMA and CSO data. Blue contours show emission integrated from -72 to $-64 \mathrm{~km} \mathrm{~s}^{-1}$ and the contour levels start from $2.6 \mathrm{Jy} \cdot \mathrm{km} \mathrm{s}^{-1}$ and increase in steps of $1.3 \mathrm{Jy} \cdot \mathrm{km} \mathrm{s}^{-1}$; red contours show emission integrated from -48 to $-42 \mathrm{~km} \mathrm{~s}^{-1}$ and the contour levels start from $3.6 \mathrm{Jy} \cdot \mathrm{km} \mathrm{s}^{-1}$ and increase in steps of $1.2 \mathrm{Jy} \cdot \mathrm{km} \mathrm{s}^{-1}$. Symbols are the same as those in Figure 2 . 


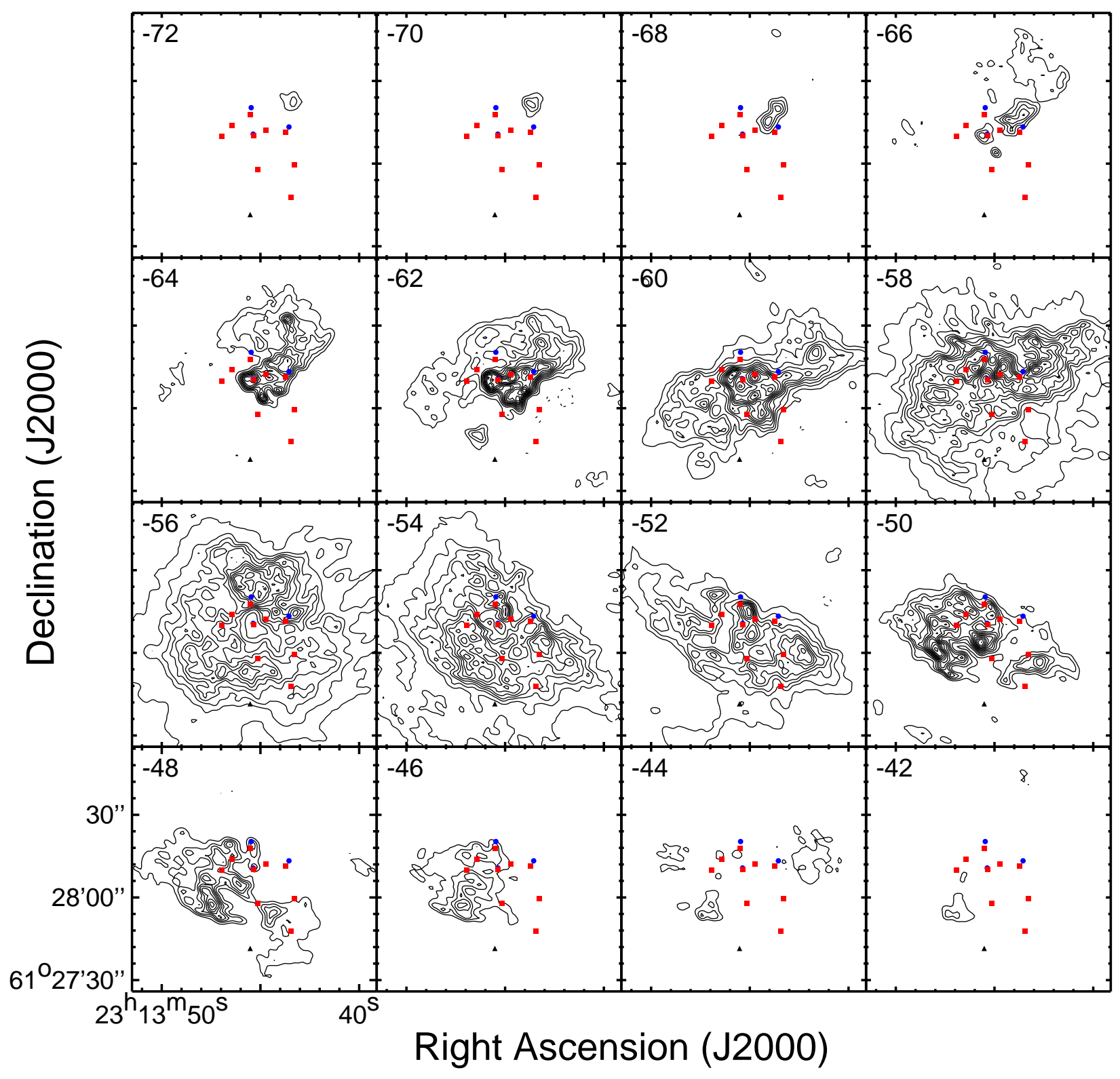

Fig. 5.- Velocity channel maps of ${ }^{13} \mathrm{CO}(2-1)$ emission obtained from the combined SMA and CSO data. The starting and spacing contour levels are $0.8 \mathrm{Jy}^{\mathrm{beam}}{ }^{-1}$ for channels from -60 to $-56 \mathrm{kms}^{-1}$ and $0.45 \mathrm{Jybeam}^{-1}$ in outer line wings. Symbols are the same as those in Figure 3 , 


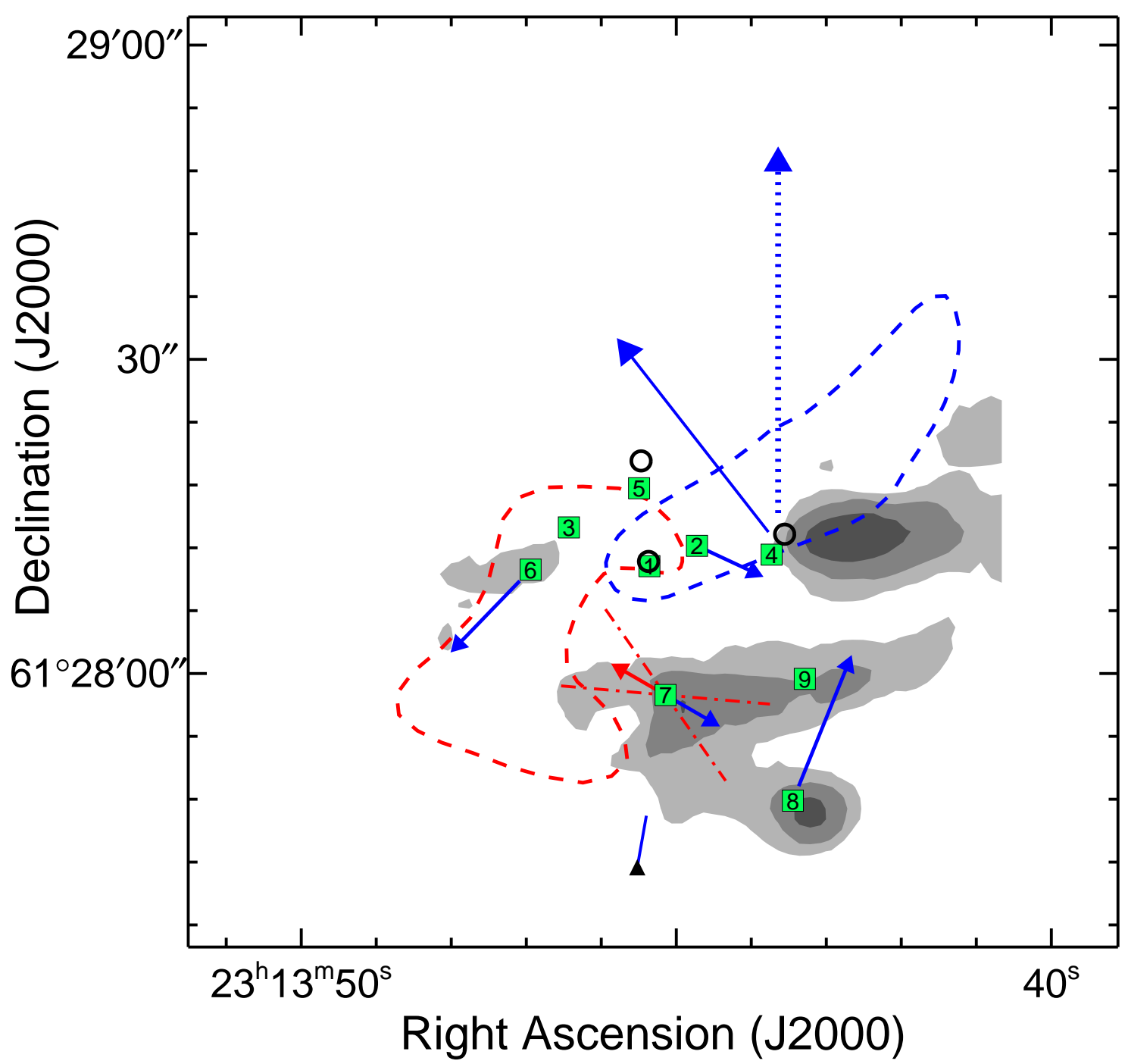

Fig. 6.- A schematic view of the multiple outflows seen in the $\mathrm{CO}$ and ${ }^{13} \mathrm{CO}$ emission. Dashed curves roughly outline the MM1/IRS 1 outflow; arrows approximately denote the orientation and extension of suggested outflows from the other cores; two dash-doted lines bisecting at MM7 highlight a bi-conical structure remarkable in ${ }^{13} \mathrm{CO}$; a $\mathrm{PA} \sim 0^{\circ}$ structure is show in a dotted arrow for its ambiguous interpretation (see Section 4.2 for details). Blue and red colors represent blue- and redshifted velocities of the structure, respectively. Other symbols are the same as those in Figure 2. The $\mathrm{NH}_{3}(2,2)$ emission is shown in filled gray contours (data from Zheng et al. 2001; see Section 4.2 for discussions on a dense clump to the west of MM4). 


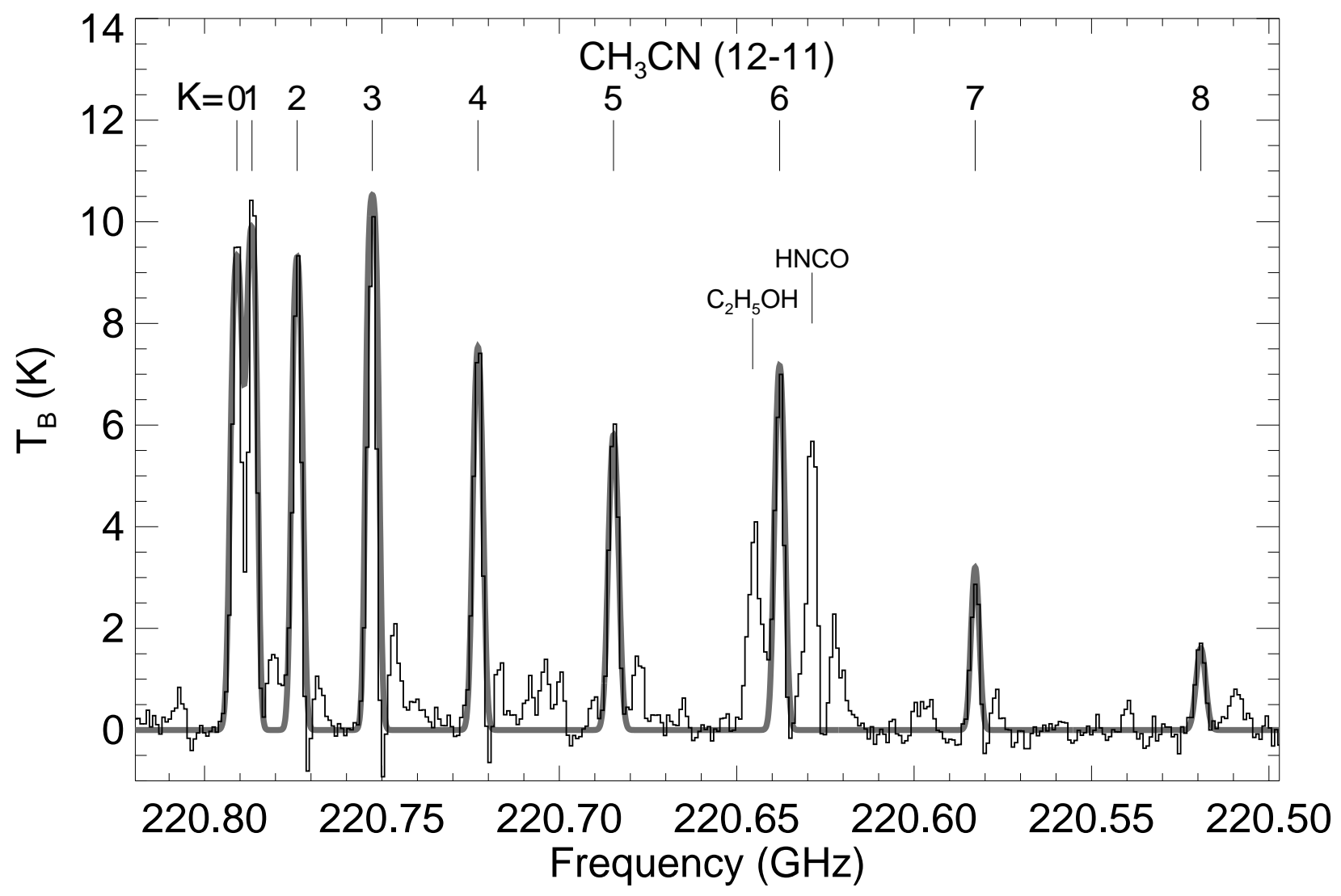

Fig. 7.- The observed spectra of $\mathrm{CH}_{3} \mathrm{CN}(12-11)$ toward MM1 in black histogram, overlaid with the best fit LTE model in thick gray. 


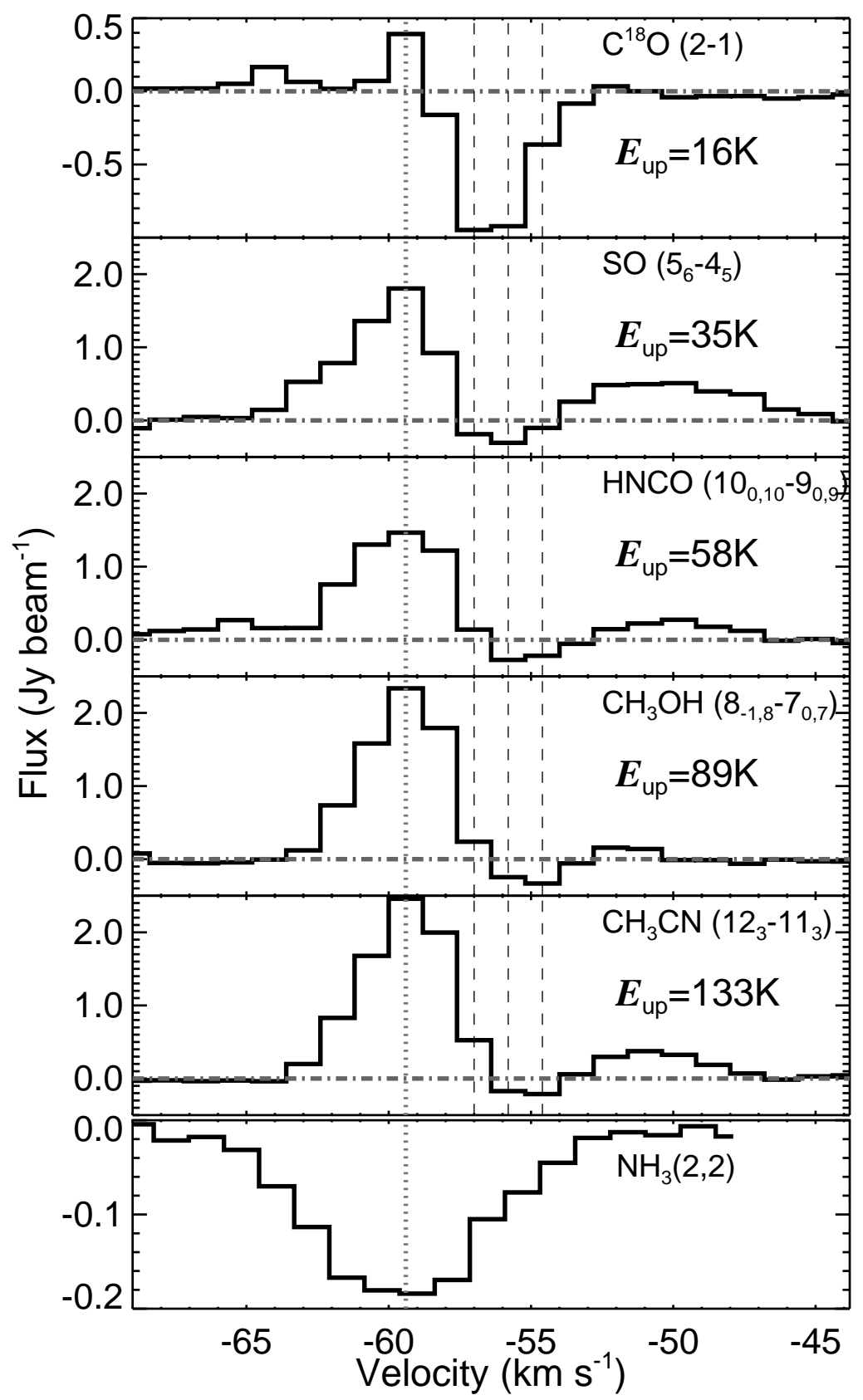

Fig. 8. - The spectra of $\mathrm{C}^{18} \mathrm{O}(2-1)$, SO $\left(5_{6}-4_{5}\right)$, $\mathrm{HNCO}\left(10_{0,10}-9_{0,9}\right), \mathrm{CH}_{3} \mathrm{OH}\left(8_{-1,8}-7_{0,7}\right)$ $E$, and $\mathrm{CH}_{3} \mathrm{CN}(12-11) \mathrm{K}=3$ toward the peak of MM1. Three dashed lines denote channel velocities at $-57,-55.8$, and $-54.6 \mathrm{~km} \mathrm{~s}^{-1}$, the peaking velocities of the absorption. The dotted gray line denotes the channel of $-59.4 \mathrm{~km} \mathrm{~s}^{-1}$, the peaking velocity of the emission in all the lines. The upper energy level of each transition is indicated in the right-hand of each panel. For comparison, the absorption in $\mathrm{NH}_{3}(2,2)$ is shown in the bottom panel (data from Zheng et al. 2001). 


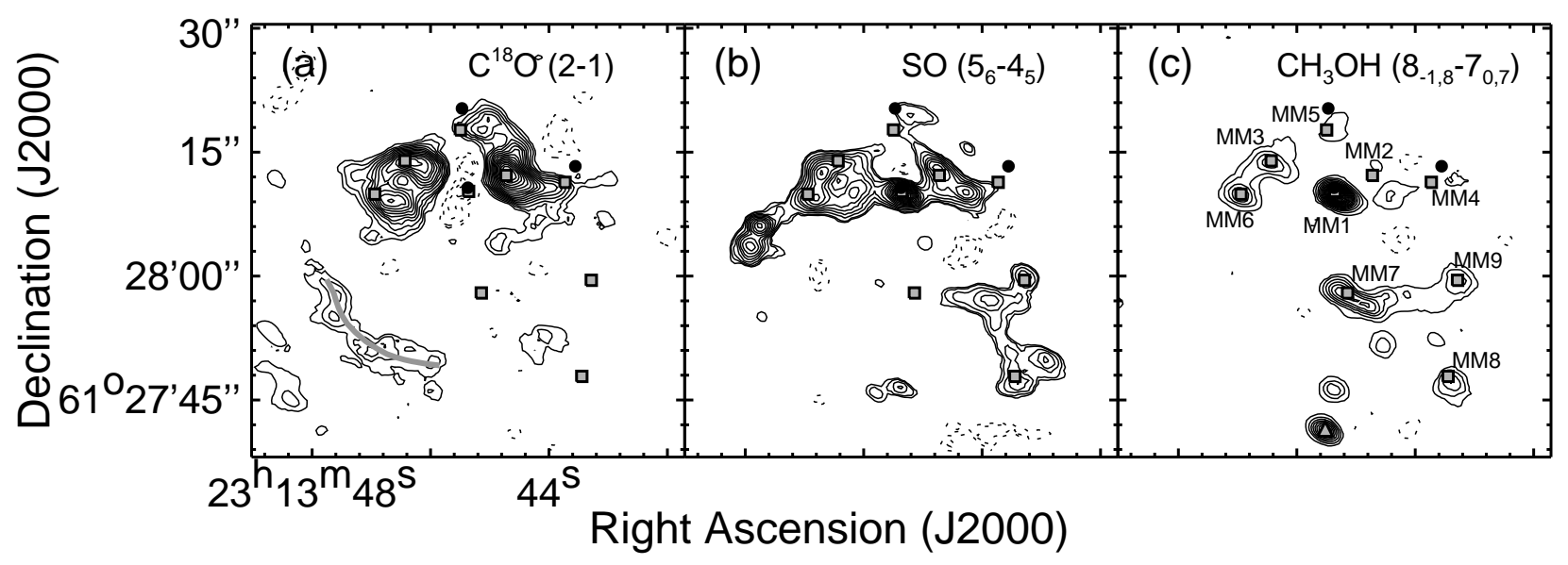

Fig. 9.- Moment-0 (velocity averaged) maps shown in contours for the lines of (a) $\mathrm{C}^{18} \mathrm{O}$ (2-1), (b) $\mathrm{SO}\left(5_{6}-4_{5}\right)$, (c) $\mathrm{CH}_{3} \mathrm{OH}\left(8_{-1,8}-7_{0,7}\right)$ E. Contour levels start from $50 \mathrm{mJy}_{\text {beam }}{ }^{-1}$ and increase with steps of $25 \mathrm{mJy}_{\text {beam }}^{-1}$ for the $\mathrm{C}^{18} \mathrm{O}$ map, start from $48 \mathrm{mJy}_{\text {beam }}{ }^{-1}$ and increase with steps of $24 \mathrm{mJy}_{\text {beam }}^{-1}$ for the SO map, and start from $24 \mathrm{mJy}^{-1}$ beam $^{-1}$ and increase with steps of $29 \mathrm{mJy}_{\text {beam }}{ }^{-1}$ for the $\mathrm{CH}_{3} \mathrm{OH}$ map. Cores MM1-9 are labeled as filled squares and for clarity, with their nomenclature shown in the $\mathrm{CH}_{3} \mathrm{OH}$ map; IRS 1-3 are denoted as three dots. 


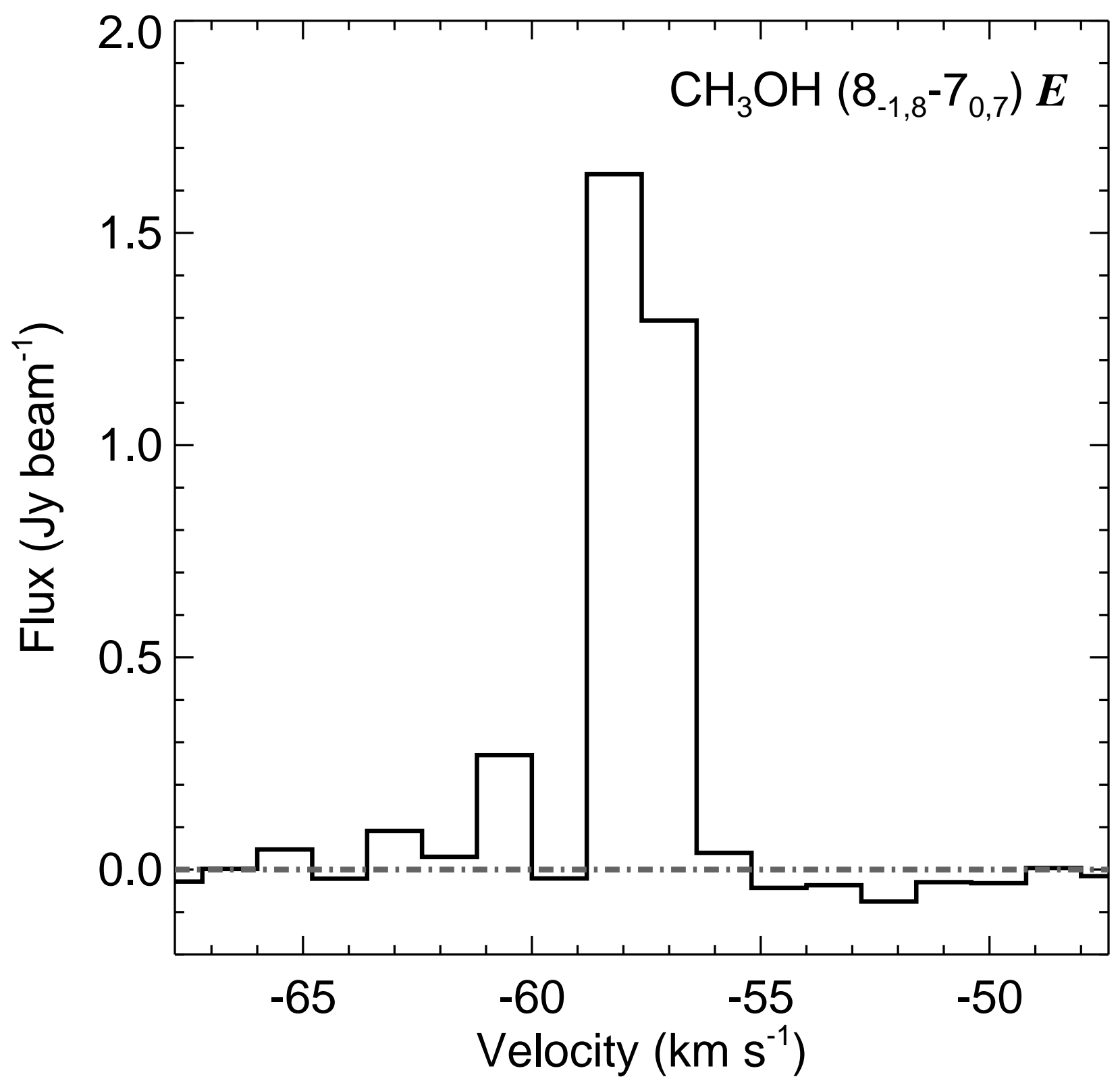

Fig. 10. - The velocity profile of the $229.8 \mathrm{GHz}$ methanol maser discovered in Figure $9 \mathrm{c}$ (see Section 3.3 .2 for details). 


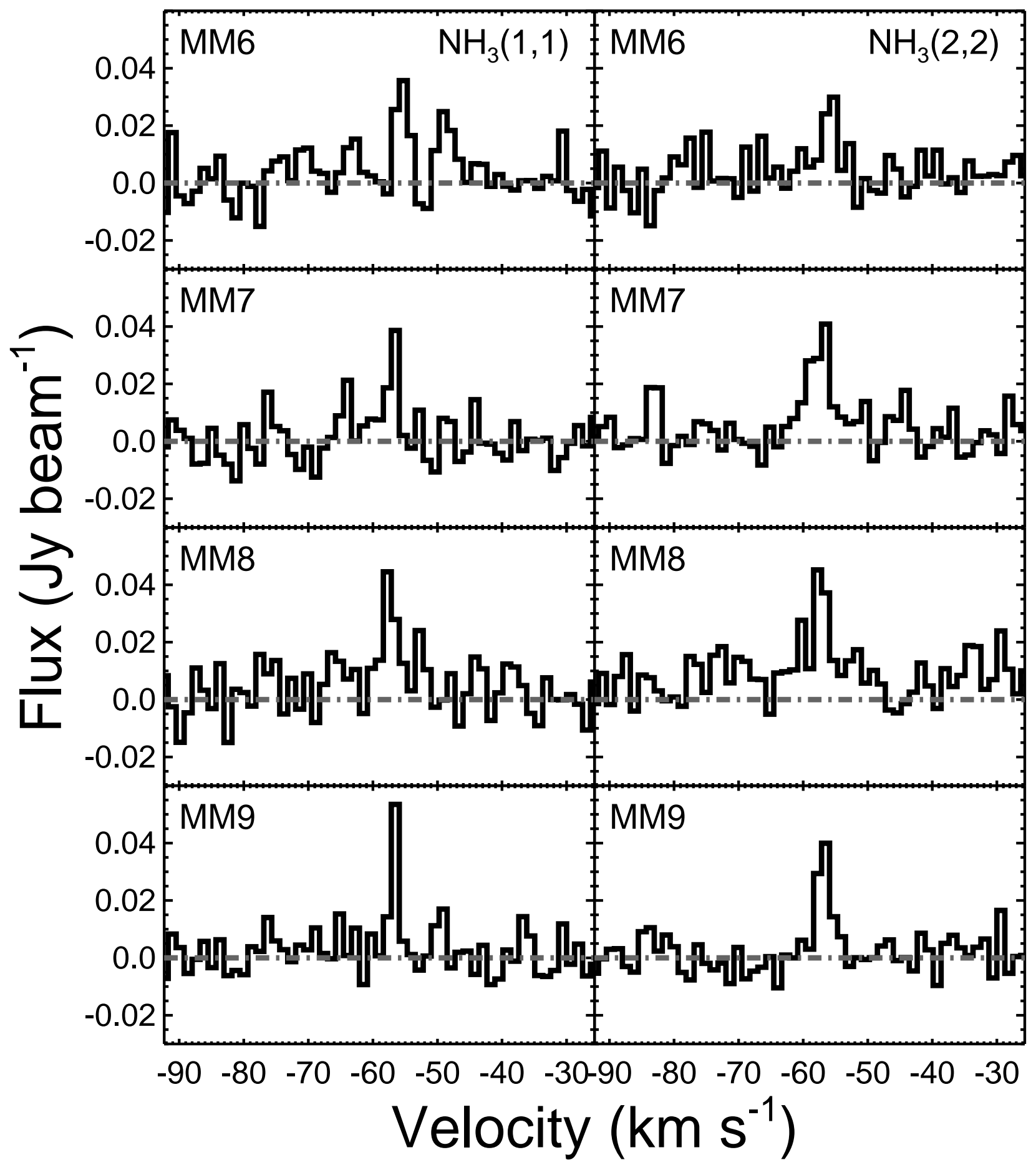

Fig. 11. - The $\mathrm{NH}_{3}(1,1)$ and (2,2) spectra in MM6-9; data taken from Zheng et al. (2001) 

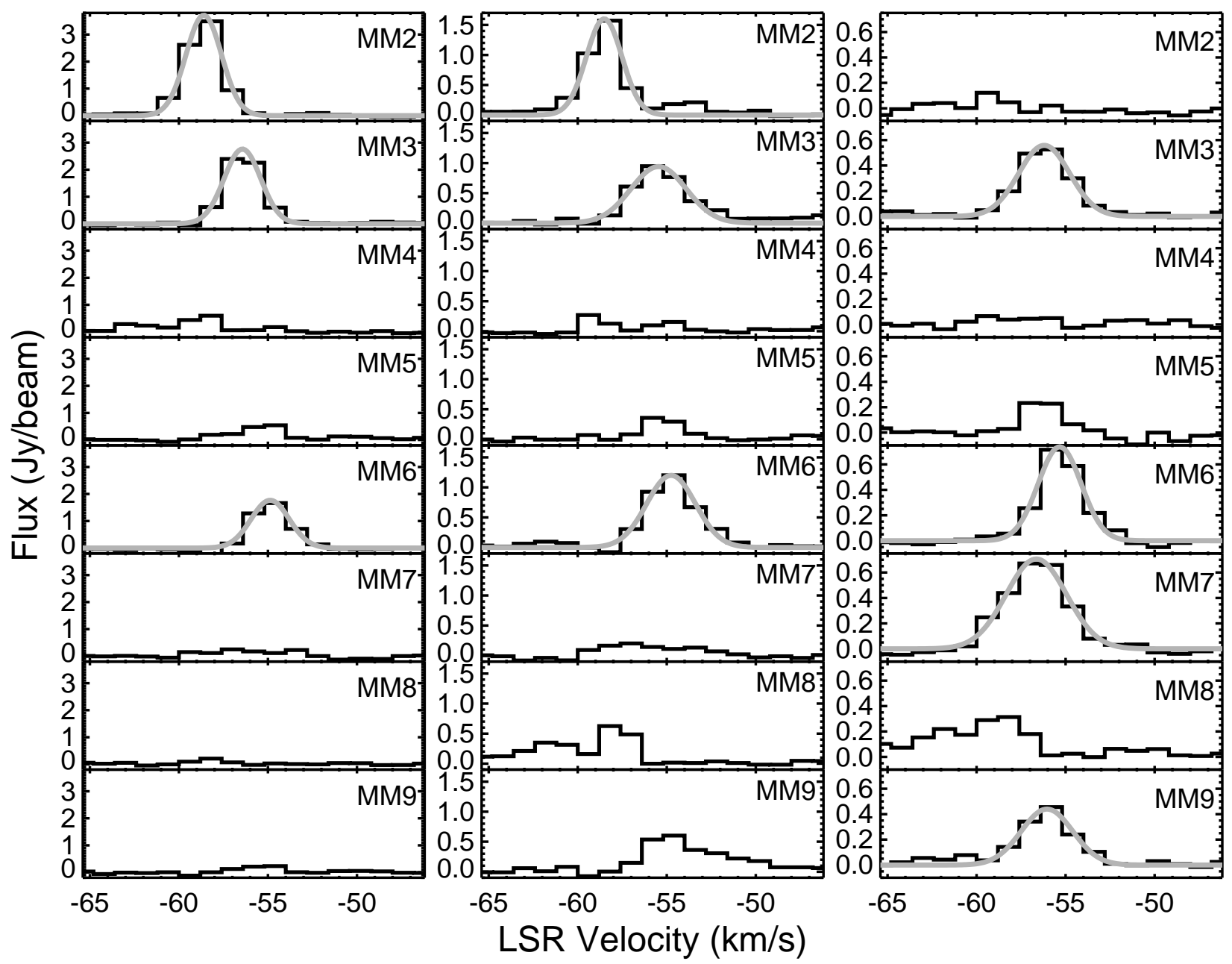

Fig. 12.- The $\mathrm{C}^{18} \mathrm{O}(2-1)$ Left), $\mathrm{SO}\left(5_{6}-_{5}\right)$ (Middle), and $\mathrm{CH}_{3} \mathrm{OH}\left(8_{-1,8^{-}} 7_{0,7}\right) E$ (Right) spectra toward the peaks of cores MM2-9 shown in histogram, with Gaussian fittings overlaid in thick gray lines. 
Table 1. Measured parameters and masses of mm continuum cores

\begin{tabular}{lccllr}
\hline \hline & $\begin{array}{c}\text { R.A. } \\
(\mathrm{J} 2000)\end{array}$ & $\begin{array}{c}\text { Decl. } \\
(\mathrm{J} 2000)\end{array}$ & $\begin{array}{c}\text { Peak Flux } \\
\left(\text { Jy beam }^{-1}\right)\end{array}$ & $\begin{array}{c}\text { Total Flux } \\
(\mathrm{Jy})\end{array}$ & $\begin{array}{c}\text { Mass }^{\mathrm{a}} \\
\left(M_{\odot}\right)\end{array}$ \\
\hline MM1 & $23^{\mathrm{h}} 13^{\mathrm{m}} 45.358^{\mathrm{s}}$ & $61^{\circ} 28^{\prime} 10.25^{\prime \prime}$ & 3.3 & 3.6 & $20.1 \pm 11.6$ \\
MM2 & $23^{\mathrm{h}} 13^{\mathrm{m}} 44.724^{\mathrm{s}}$ & $61^{\circ} 28^{\prime} 12.19^{\prime \prime}$ & 0.18 & 0.37 & $24.4 \pm 12.7$ \\
MM3 & $23^{\mathrm{h}} 13^{\mathrm{m}} 46.432^{\mathrm{s}}$ & $61^{\circ} 28^{\prime} 13.95^{\prime \prime}$ & 0.17 & 0.31 & $20.5 \pm 10.7$ \\
MM4 & $23^{\mathrm{h}} 13^{\mathrm{m}} 43.729^{\mathrm{s}}$ & $61^{\circ} 28^{\prime} 11.35^{\prime \prime}$ & 0.11 & 0.22 & $14.5 \pm 7.8$ \\
MM5 & $23^{\mathrm{h}} 13^{\mathrm{m}} 45.496^{\mathrm{s}}$ & $61^{\circ} 28^{\prime} 17.69^{\prime \prime}$ & 0.11 & 0.30 & $19.8 \pm 10.6$ \\
MM6 & $23^{\mathrm{h}} 13^{\mathrm{m}} 46.942^{\mathrm{s}}$ & $61^{\circ} 28^{\prime} 09.92^{\prime \prime}$ & 0.10 & 0.15 & $9.1 \pm 5.9$ \\
MM7 & $23^{\mathrm{h}} 13^{\mathrm{m}} 45.143^{\mathrm{s}}$ & $61^{\circ} 27^{\prime} 57.95^{\prime \prime}$ & 0.082 & 0.12 & $5.2 \pm 2.9$ \\
MM8 & $23^{\mathrm{h}} 13^{\mathrm{m}} 43.446^{\mathrm{s}}$ & $61^{\circ} 27^{\prime} 47.86^{\prime \prime}$ & 0.067 & 0.10 & $4.7 \pm 3.0$ \\
MM9 & $23^{\mathrm{h}} 13^{\mathrm{m}} 43.286^{\mathrm{s}}$ & $61^{\circ} 27^{\prime} 59.49^{\prime \prime}$ & 0.049 & 0.088 & $6.2 \pm 3.0$ \\
\hline
\end{tabular}

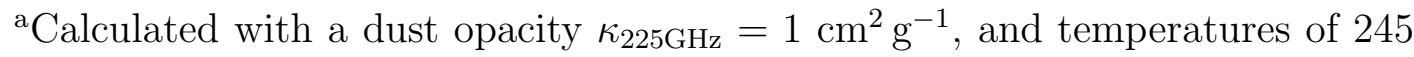
$\mathrm{K}$ for MM1, $40 \mathrm{~K}$ for MM2-5, and 43, 58, 54, $38 \mathrm{~K}$ for MM6-9; see the discussion in Section 3.1 for details about the mass uncertainties. 
Table 2. Derived outflow parameters

\begin{tabular}{cccccc}
\hline \hline $\begin{array}{c}M_{\text {out }}{ }^{\mathrm{a}} \\
\left(M_{\odot}\right)\end{array}$ & $\begin{array}{c}P_{\text {out }}{ }^{\mathrm{b}} \\
\left(M_{\odot} \mathrm{km} \mathrm{s}^{-1}\right)\end{array}$ & $\begin{array}{c}E_{\text {out }} \\
(\mathrm{erg})\end{array}$ & $\begin{array}{c}t_{\text {dyn }} \\
(\mathrm{yr})\end{array}$ & $\begin{array}{c}\dot{M}_{\text {out }} \\
\left(M_{\odot} \mathrm{yr}^{-1}\right)\end{array}$ & $\begin{array}{c}\dot{P}_{\text {out }} \\
\left(M_{\odot} \mathrm{km} \mathrm{s}^{-1} \mathrm{yr}^{-1}\right)\end{array}$ \\
\hline 50 & $4.6 \times 10^{2}$ & $4.9 \times 10^{46}$ & $2 \times 10^{4}$ & $2.5 \times 10^{-3}$ & $2.3 \times 10^{-2}$ \\
\hline
\end{tabular}

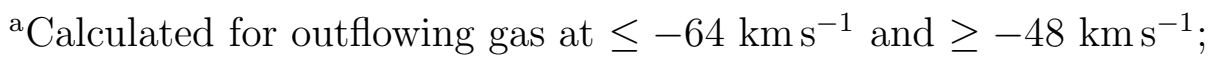

${ }^{\mathrm{b}}$ Without correcting for an unknown inclination angle; this affects all the parameters except $M_{\text {out }}$; 
Table 3. Molecular lines detected in MM1 ${ }^{\mathrm{a}}$

\begin{tabular}{|c|c|c|c|}
\hline $\begin{array}{l}\text { Frequency }^{\mathrm{b}} \\
\quad(\mathrm{GHz})\end{array}$ & Line $^{c}$ & $\begin{array}{l}E_{\mathrm{up}} \\
(\mathrm{K})\end{array}$ & $\begin{array}{c}\text { Peak }^{\mathrm{d}} \\
\left(\mathrm{Jy} \mathrm{beam}^{-1}\right)\end{array}$ \\
\hline 219.560357 & $\mathrm{C}^{18} \mathrm{O} 2-1$ & 16 & 0.52 \\
\hline 219.949442 & $\mathrm{SO} 5_{6}-4_{5}$ & 35 & 2.45 \\
\hline 229.347628 & $\mathrm{SO}_{2} 11_{5,7}-12_{4,8}$ & 122 & 0.29 \\
\hline 231.060983 & OCS 19-18 & 111 & 2.55 \\
\hline 219.908525 & $\mathrm{H}_{2}^{13} \mathrm{CO} 3_{1,2}-2_{1,1}$ & 33 & 0.81 \\
\hline 219.656710 & HNCO $10_{3,8(7)}-9_{3,7(6)}$ & 448 & 0.56 \\
\hline 219.733850 & HNCO $10_{2,11(10)(9)}-9_{2,10(9)(8)}$ & 231 & 1.33 \\
\hline 219.798320 & HNCO $10_{0,11(10)(9)}-9_{0,10(9)(8)}$ & 58 & 1.53 \\
\hline 220.585200 & HNCO $10_{1,11(10)(9)}-9_{1,10(9)(8)}$ & 102 & 1.46 \\
\hline 220.038072 & $\mathrm{HCOOH} 10_{0,10}-9_{0,9}$ & 59 & 0.66 \\
\hline 220.178196 & $\mathrm{CH}_{2} \mathrm{CO} 11_{1,11}-10_{0,10}$ & 77 & 0.98 \\
\hline 219.983675 & $\mathrm{CH}_{3} \mathrm{OH} 25_{3,22}-24_{4,20} E$ & 803 & 0.75 \\
\hline 219.993658 & $\mathrm{CH}_{3} \mathrm{OH} 23_{5,19}-22_{6,17} E$ & 777 & 0.64 \\
\hline 220.078561 & $\mathrm{CH}_{3} \mathrm{OH} 8_{0,8}-7_{1,6} E$ & 97 & 2.92 \\
\hline 220.886784 & $\mathrm{CH}_{3} \mathrm{OH} 31_{-2}-31_{1}$ & 1182 & 0.45 \\
\hline 229.589056 & $\mathrm{CH}_{3} \mathrm{OH} 15_{4,11}-16_{3,13} E$ & 375 & 4.59 \\
\hline 229.758756 & $\mathrm{CH}_{3} \mathrm{OH} 8_{-1,8}-7_{0,7} E$ & 89 & 2.86 \\
\hline 229.864121 & $\mathrm{CH}_{3} \mathrm{OH} 19_{5,15}-20_{4,16} A+$ & 579 & 1.79 \\
\hline 229.939095 & $\mathrm{CH}_{3} \mathrm{OH} 19_{5,14}-20_{4,17} A-$ & 579 & 1.93 \\
\hline $230.027047^{\mathrm{e}}$ & $\mathrm{CH}_{3} \mathrm{OH} 3_{-2,2}-4_{-1,4} E$ & 40 & 3.36 \\
\hline $230.368763^{\mathrm{f}}$ & $\mathrm{CH}_{3} \mathrm{OH} 22_{4,18}-21_{5,17} E$ & 684 & 1.56 \\
\hline $220.362774^{\mathrm{g}}$ & ${ }^{13} \mathrm{CH}_{3} \mathrm{OH} 17_{-3,15}-18_{0,18}$ & 410 & 0.41 \\
\hline 220.475807 & $\mathrm{CH}_{3} \mathrm{CN} 12_{8}-11_{8}$ & 526 & 0.39 \\
\hline 220.539324 & $\mathrm{CH}_{3} \mathrm{CN} 12_{7}-11_{7}$ & 419 & 0.71 \\
\hline 220.594423 & $\mathrm{CH}_{3} \mathrm{CN} 12_{6}-11_{6}$ & 326 & 1.87 \\
\hline 220.641084 & $\mathrm{CH}_{3} \mathrm{CN} 12_{5}-11_{5}$ & 248 & 1.66 \\
\hline 220.679287 & $\mathrm{CH}_{3} \mathrm{CN} 12_{4}-11_{4}$ & 183 & 2.07 \\
\hline 220.709017 & $\mathrm{CH}_{3} \mathrm{CN} 12_{3}-11_{3}$ & 133 & 2.98 \\
\hline 220.730261 & $\mathrm{CH}_{3} \mathrm{CN} 12_{2}-11_{2}$ & 98 & 2.79 \\
\hline 220.743011 & $\mathrm{CH}_{3} \mathrm{CN} 12_{1}-11_{1}$ & 76 & 3.05 \\
\hline
\end{tabular}


Table 3-Continued

\begin{tabular}{|c|c|c|c|}
\hline $\begin{array}{l}\text { Frequency }^{\mathrm{b}} \\
\quad(\mathrm{GHz})\end{array}$ & Line $^{c}$ & $\begin{array}{l}E_{\text {up }} \\
(\mathrm{K})\end{array}$ & $\begin{array}{c}\text { Peak }^{\mathrm{d}} \\
\left(\mathrm{Jy} \mathrm{beam}^{-1}\right)\end{array}$ \\
\hline 220.747261 & $\mathrm{CH}_{3} \mathrm{CN} 12_{0}-11_{0}$ & 69 & 2.78 \\
\hline 219.268715 & $\mathrm{CH}_{3} \mathrm{CHO} 31_{5,27}-32_{2,30}$ & 517 & 0.43 \\
\hline 230.301880 & $\mathrm{CH}_{3} \mathrm{CHO} 12_{2,11}-11_{2,10}$ & 81 & 0.54 \\
\hline 219.411703 & $\mathrm{CH}_{3} \mathrm{OCHO} 18_{10,8}-17_{10,7}$ & 355 & 0.39 \\
\hline 219.763947 & $\mathrm{CH}_{3} \mathrm{OCHO} 18_{9,9}-17_{9,8}$ & 342 & 0.64 \\
\hline 219.822126 & $\mathrm{CH}_{3} \mathrm{OCHO} 18_{10,9(8)}-17_{10,8(7)}$ & 355 & 0.41 \\
\hline 220.030339 & $\mathrm{CH}_{3} \mathrm{OCHO} 18_{9,10}-17_{9,9}$ & 342 & 0.57 \\
\hline 220.166888 & $\mathrm{CH}_{3} \mathrm{OCHO} 17_{4,13}-16_{4,12}$ & 103 & 1.26 \\
\hline 220.190285 & $\mathrm{CH}_{3} \mathrm{OCHO} 17_{4,13}-16_{4,12}$ & 103 & 1.32 \\
\hline 220.258096 & $\mathrm{CH}_{3} \mathrm{OCHO} 18_{8,10^{-}}-17_{8,9}$ & 331 & 0.41 \\
\hline 220.369877 & $\mathrm{CH}_{3} \mathrm{OCHO} 18_{8,10}-17_{8,9}$ & 331 & 0.52 \\
\hline 220.913955 & $\mathrm{CH}_{3} \mathrm{OCHO} 18_{7,12}-17_{7,11}$ & 321 & 0.36 \\
\hline 220.946352 & $\mathrm{CH}_{3} \mathrm{OCHO} 18_{7,11}-17_{7,10}$ & 321 & 0.34 \\
\hline 220.977984 & $\mathrm{CH}_{3} \mathrm{OCHO} 18_{15,3}-17_{15,2}$ & 250 & 0.38 \\
\hline 220.985330 & $\mathrm{CH}_{3} \mathrm{OCHO} 18_{7,11}-17_{7,10}$ & 321 & 0.45 \\
\hline 220.998335 & $\mathrm{CH}_{3} \mathrm{OCHO} 18_{15,4}-17_{15,3}$ & 250 & 0.52 \\
\hline 221.047791 & $\mathrm{CH}_{3} \mathrm{OCHO} 18_{14,5(4)}-17_{14,4(3)}$ & 231 & 0.50 \\
\hline 221.066933 & $\mathrm{CH}_{3} \mathrm{OCHO} 18_{14,5}-17_{14,4}$ & 231 & 0.36 \\
\hline 221.141129 & $\mathrm{CH}_{3} \mathrm{OCHO} 18_{13,6(5)}-17_{13,5(4)}$ & 213 & 0.97 \\
\hline 229.405021 & $\mathrm{CH}_{3} \mathrm{OCHO} 18_{3,15}-17_{3,14}$ & 111 & 1.44 \\
\hline 229.420342 & $\mathrm{CH}_{3} \mathrm{OCHO} 18_{3,15}-17_{3,14}$ & 111 & 1.45 \\
\hline 230.293951 & $\mathrm{CH}_{3} \mathrm{OCHO} 22_{9,13}-22_{8,14}$ & 204 & 0.75 \\
\hline $230.315800^{\mathrm{h}}$ & $\mathrm{CH}_{3} \mathrm{OCHO} 22_{9,14}-22_{8,15}$ & 204 & 0.69 \\
\hline 230.878810 & $\mathrm{CH}_{3} \mathrm{OCHO} 18_{4,14}-17_{14,13}$ & 301 & 0.45 \\
\hline 220.601927 & $\mathrm{C}_{2} \mathrm{H}_{5} \mathrm{OH} 13_{1,13}-12_{0,12}$ & 75 & 1.08 \\
\hline $229.491131^{\mathrm{i}}$ & $\mathrm{C}_{2} \mathrm{H}_{5} \mathrm{OH} 17_{5,12}-17_{4,13}$ & 160 & 0.66 \\
\hline 229.818039 & $\mathrm{C}_{2} \mathrm{H}_{5} \mathrm{OH} 26_{5,22}-26_{4,23}$ & 385 & 0.43 \\
\hline 230.177998 & $\mathrm{C}_{2} \mathrm{H}_{5} \mathrm{OH} 45_{2,44}-44_{3,43}$ & 894 & 0.41 \\
\hline 230.230743 & $\mathrm{C}_{2} \mathrm{H}_{5} \mathrm{OH} 13_{2,11}-12_{2,10}$ & 143 & 0.66 \\
\hline $230.473005^{\mathrm{j}}$ & $\mathrm{C}_{2} \mathrm{H}_{5} \mathrm{OH} 18_{3,16}-17_{4,14}$ & 215 & 0.38 \\
\hline
\end{tabular}


Table 3-Continued

\begin{tabular}{llrr}
\hline \hline $\begin{array}{c}\text { Frequency } \\
(\mathrm{GHz})\end{array}$ & \multicolumn{1}{c}{ Line $^{\mathrm{c}}$} & $\begin{array}{c}E_{\text {up }} \\
(\mathrm{K})\end{array}$ & $\begin{array}{c}\text { Peak }^{\mathrm{d}} \\
\left(\mathrm{Jy} \mathrm{beam}^{-1}\right)\end{array}$ \\
\hline 230.672554 & $\mathrm{C}_{2} \mathrm{H}_{5} \mathrm{OH} 13_{2,11}-12_{2,10}$ & 139 & 0.66 \\
$230.793764^{\mathrm{k}}$ & $\mathrm{C}_{2} \mathrm{H}_{5} \mathrm{OH} 6_{5,1}-5_{4,1}$ & 105 & 0.53 \\
230.953778 & $\mathrm{C}_{2} \mathrm{H}_{5} \mathrm{OH} \mathrm{16} 6_{5,11}-16_{4,12}$ & 146 & 0.75 \\
230.991374 & $\mathrm{C}_{2} \mathrm{H}_{5} \mathrm{OH} 14_{0,14}-13_{1,13}$ & 86 & 1.08 \\
220.893110 & $\mathrm{CH}_{3} \mathrm{OCH}_{3} 23_{4,20}-23_{3,21}$ & 275 & 0.40 \\
230.141374 & $\mathrm{CH}_{3} \mathrm{OCH}_{3} 25_{4,22}-25_{3,22}$ & 319 & 0.51 \\
$229.234^{\mathrm{m}}$ & - & - & 0.29 \\
$230.578^{\mathrm{m}}$ & - & - & 0.65 \\
\hline
\end{tabular}

${ }^{\mathrm{a}} \mathrm{We}$ list lines (excluding $\mathrm{CO}$ and ${ }^{13} \mathrm{CO} 2-1$ ) detected above $6 \sigma\left(0.21 \mathrm{Jy}^{-1}\right.$ beam $\left.^{-1}\right)$;

${ }^{\mathrm{b}}$ Rest frequencies obtained from the JPL catalog at http://spec.jpl.nasa.gov/ftp/pub/catalog/catform.html;

${ }^{\mathrm{c}}$ Quantum numbers in parentheses denote degeneracy, for instance, HNCO $10_{3,8(7)}-9_{3,7(6)}$ represents HNCO $10_{3,8}-9_{3,7}$ and $\mathrm{HNCO} 10_{3,7}-9_{3,6}$;

${ }^{\mathrm{d}}$ Derived with the MIRIAD task MAXFIT;

${ }^{\mathrm{e}}$ Under LTE, the peak flux is unexpectedly higher than other $\mathrm{CH}_{3} \mathrm{OH}$ lines (e.g., $\mathrm{CH}_{3} \mathrm{OH}\left(8_{-1,8}-7_{0,7}\right) E$ ), which may be partly due to blending with $\mathrm{C}_{3} \mathrm{H}_{7} \mathrm{CN}\left(52_{6,46}-51_{6,45}\right)$, $\nu_{\mathrm{o}}=230.027116 \mathrm{GHz}$ and $\mathrm{C}_{2} \mathrm{H}_{3} \mathrm{CN}\left(4_{3,1}-4_{2,2}\right), \nu_{\circ}=230.026216$ $\mathrm{GHz}$;

${ }^{\mathrm{f}}$ Blended with $\mathrm{CH}_{3} \mathrm{OCH}_{3} \quad\left(28_{5,23}-27_{6,22}\right), \quad \nu_{\circ}=230.368178$ $\mathrm{GHz}$;

${ }^{g}$ Not listed in the JPL catalog, but included in Cologne Database for Molecular Spectroscopy; 
${ }^{\text {h}}$ Blended with $\mathrm{CH}_{3} \mathrm{CHO} \quad\left(12_{2,11}-11_{2,10}\right), \quad \nu_{\circ}=230.315740$ $\mathrm{GHz}$;

${ }^{i}$ Blended with $\mathrm{CH}_{3} \mathrm{OCHO}\left(31_{5,27}-31_{4,28}\right), \quad \nu_{\circ}=229.492067$ $\mathrm{GHz}$;

jBlended with $\mathrm{CH}_{3} \mathrm{OCH}_{3}\left(10_{8,3}-11_{7,4}\right), \nu_{\circ}=230.473591 \mathrm{GHz}$;

${ }^{\mathrm{k}}$ Blended with $\mathrm{C}_{2} \mathrm{H}_{5} \mathrm{OH}\left(6_{5,2}-5_{4,2}\right), \nu_{\circ}=230.793864 \mathrm{GHz}$;

${ }^{1}$ Probably blended with $\mathrm{C}_{3} \mathrm{H}_{7} \mathrm{CN} \quad\left(36_{6,31}-35_{5,30}\right)$, $\nu_{\circ}=230.142063 \mathrm{GHz}$;

${ }^{\mathrm{m}}$ Not identified. 